\title{
Article
}

\section{On Single-Valued Neutrosophic Closure Spaces}

\author{
Fahad Alsharari 1,2 (D) \\ 1 Department of Mathematics, College of Science and Human Studies, Majmaah University, \\ Hotat Sudair 11982, Saudi Arabia; f.alsharari@mu.edu.sa \\ 2 Department of Mathematics, College of Science and Arts, Jouf University, Gurayat, 77455, Saudi Arabia
}

check for

updates

Citation: Alsharari, F. On Single-Valued Neutrosophic Closure Spaces. Symmetry 2021, 13, 1508. https://doi.org/10.3390/sym13081508

Academic Editor: Sergei D. Odintsov

Received: 30 June 2021

Accepted: 11 August 2021

Published: 17 August 2021

Publisher's Note: MDPI stays neutral with regard to jurisdictional claims in published maps and institutional affiliations.

\begin{abstract}
This paper aims to mark out new terms of single-valued neutrosophic notions in a Šostak sense called single-valued neutrosophic semi-closure spaces. To achieve this, notions such as $\beta £$-closure operators and $\beta £$-interior operators are first defined. More precisely, these proposed contributions involve different terms of single-valued neutrosophic continuous mappings called single-valued neutrosophic (almost $\beta £$, faintly $\beta £$, weakly $\beta £$ ) and $\beta £$-continuous. Finally, for the purpose of symmetry, we define the single-valued neutrosophic upper, single-valued neutrosophic lower and single-valued neutrosophic boundary sets of a rough single-valued neutrosophic set $\alpha_{n}$ in a single-valued neutrosophic approximation space $(\tilde{\mathcal{F}}, \delta)$. Based on $\alpha_{n}$ and $\delta$, we also introduce the single-valued neutrosophic approximation interior operator int $\mathrm{\alpha}_{\alpha_{n}}^{\delta}$ and the single-valued neutrosophic approximation closure operator $\mathrm{Cl}_{\alpha_{n}}^{\delta}$.
\end{abstract}

Keywords: single-valued neutrosophic $\beta E$-closure operators; $\beta £$-interior operators; single-valued neutrosophic almost $\beta £$-continuous; faintly $\beta £$-continuous; weakly $\beta £$-continuous; $\beta £$-continuous; single-valued neutrosophic approximation space; approximation interior operator; approximation closure operator

\section{Introduction}

Neutrosophic set theory has a very powerful influence given that is a recent section of philosophy that is presented as the study of origin, nature and scope of neutralities. The idea of neutrosophy is initiated by Smarandache [1] in 1999 as a new mathematical approach that corresponds to degree of indeterminacy (uncertainty, etc.). Moreover, the soft set theory was successfully applied to several directions, such as smoothness of functions and architecture-based neuro-linguistic programming (NLP) in the papers of Bakbak et al. [2] and Mishra et al. [3]. The concept of continuous mappings plays a crucial role in many branches of mathematics, such as, fuzzy set theory, algebra and quantum gravity (see [4]). El-Naschie also has shown that both string theory and $\varepsilon^{\infty}$ theory are kind of some applications in quantum particle physics especially in relation to heterotic strings and were influenced by the fuzzy topology in Šostak sense. [5].

In current times, the theory of neutrosophy has been recycled at various junctions of mathematics. More precisely, this theory has made an exceptional advancement in the field of topological spaces. Salama et al. [6-8] dispatched their works of neutrosophic topological spaces, following the method of Chang [9] in the situation of fuzzy topological spaces $(\tilde{\mathcal{F}}, \tau)$. Afterward, Hur et al. [10,11] presented NSet(H) and NCSet. Smarandache [12] defined the idea of neutrosophic topology on the non-standard interval. One can simply detect that the fuzzy topology familiarized by Chang is a crisp group of fuzzy subsets.

Šostak [13] determined that Chang's style is crisp in nature and so he redefined the idea of fuzzy topology, frequently mentioned as smooth fuzzy topology, as a mapping from the group of all fuzzy subsets of $\tilde{\mathcal{F}}$ to $[0,1]$. Fang Jin-ming et al. and Zahran et al. $[14,15]$ discussed the notion of foundation as a function from an appropriate collection of fuzzy subsets of $X$ to $[0,1]$. Saber et al. [16] found a parallel theory in the context fuzzy ideal topological space. 
Wang [17], in 2010, established the idea of a single-valued neutrosophic set. In 2016, Gayyar [18] presented the notion of fuzzy neutrosophic topological spaces in a Šostak sense. The concept of the foundation for an ordinary single-valued neutrosophic topology was explored by Kim [19]. Several authors [20-25] posted their efforts for the idea of single-valued neutrosophic topological spaces $\left(\tilde{\mathcal{F}}, \tau^{\tilde{\sigma} \tilde{\sigma}}\right)$. Others focusing their works on single valued neutrosophic relations, see [26,27]. Last but not least, in the sense that not only the objects are fuzzified, but also the axiomatics, the single-valued neutrsophic ideal theory was introduced in 1985 by Šostak [13] as a generalization of classical topological structures and as an extension of both crisp topology and Changs fuzzy topology.

In this article, preliminaries of single-value neutrosophic sets and single-valued neutrosophic topology are reviewed in Section 2. In Section 3, we define the notions of a singlevalued neutrosophic semi-closure space. Some of their characteristic properties are considered. Further, we present and explore the properties and characterizations of the single-

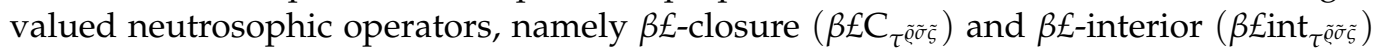
in the single-valued neutrosophic ideal topological space $\left(\tilde{\mathcal{F}}, \tau^{\tilde{\varrho} \tilde{\sigma} \tilde{\zeta}}, £^{\tilde{\varrho} \tilde{\sigma} \tilde{\zeta}}\right)$. The concepts of single-valued neutrosophic (almost, faintly, weakly) $\beta €$-continuous mappings are introduced and studied in Section 4. In Section 5, we introduce a new improved single-valued neutrosophic lower and single-valued neutrosophic upper sets by which we obtain a more reliable single-valued neutrosophic boundary region set of a single-valued neutrosophic set $\alpha_{n}$. From these single-valued neutrosophic lower and fuzzy upper sets, we define new single-valued neutrosophic interior and single-valued neutrosophic closure operators associated with a specific single-valued neutrosophic set $\alpha_{n} \in \zeta^{\tilde{\mathcal{F}}}$.

\section{Preliminaries}

This section is devoted to bring a complete survey, some previous studies and important related notions to this work. Let us have a fixed universe $\tilde{\mathcal{F}}$ to be a finite set of objects and $\zeta$ a closed unit interval $[0,1]$. We will also let $\zeta^{\mathcal{F}}$ to denote the set of all single-valued neutrosophic subsets of $\tilde{\mathcal{F}}$.

Definition 1 ([12]). Let $\tilde{\mathcal{F}}$ be a non-empty set. A neutrosophic set (briefly, $\mathcal{N S})$ in $\tilde{\mathcal{F}}$ is an object having the form

$$
\alpha_{n}=\left\{\left\langle v, \tilde{\varrho}_{\alpha_{n}}(v), \tilde{\sigma}_{\sigma_{n}}(v), \tilde{\zeta}_{\alpha_{n}}(v)\right\rangle: v \in \tilde{\mathcal{F}}\right\}
$$

where

$$
\tilde{\varrho}: \tilde{\mathcal{F}} \rightarrow\rfloor^{-} 0,1^{+}\lfloor, \tilde{\sigma}: \tilde{\mathcal{F}} \rightarrow\rfloor^{-} 0,1^{+}\lfloor, \tilde{\zeta}: \tilde{\mathcal{F}} \rightarrow\rfloor^{-} 0,1^{+}\lfloor,
$$

and

$$
{ }^{-} 0 \leq \tilde{\varrho}_{\alpha_{n}}(v)+\tilde{\sigma}_{\alpha_{n}}(v)+\tilde{\zeta}_{\alpha_{n}}(v) \leq 3^{+}
$$

represent the degree of membership $\left(\tilde{\varrho}_{\alpha_{n}}\right)$, the degree of indeterminacy $\left(\tilde{\sigma}_{\alpha_{n}}\right)$ and the degree of non-membership $\left(\tilde{\zeta}_{\alpha_{n}}\right)$, respectively, of any $v \in \tilde{\mathcal{F}}$ to the set $\alpha_{n}$.

Definition 2 ([17]). Suppose that $\tilde{\mathcal{F}}$ is a universal set a space of points (objects), with a generic element in $\tilde{\mathcal{F}}$ denoted by $v$. Then, $\alpha_{n}$ is called a single-valued neutrosophic set (briefly, $\left.\mathcal{S} \mathcal{V} \mathcal{N} \mathcal{S}\right)$ in $\tilde{\mathcal{F}}$, if $\alpha_{n}$ has the form

$$
\alpha_{n}=\left\{\left\langle v, \tilde{\varrho}_{\alpha_{n}}(v), \tilde{\sigma}_{\alpha_{n}}(v), \tilde{\zeta}_{\alpha_{n}}(v)\right\rangle: v \in \tilde{\mathcal{F}}\right\}
$$

Now, $\tilde{\varrho}_{\alpha_{n}}, \tilde{\sigma}_{\sigma_{n}}, \tilde{\zeta}_{\alpha_{n}}$ indicate the degree of non-membership, the degree of indeterminacy and the degree of membership, respectively, of any element $v \in \tilde{\mathcal{F}}$ to the set $\alpha_{n}$.

Definition 3 ([17]). Let $\alpha_{n}=\left\{\left\langle v, \tilde{\varrho}_{\alpha_{n}}(v), \tilde{\sigma}_{\sigma_{n}}(v), \tilde{\zeta}_{\alpha_{n}}(v)\right\rangle: v \in \tilde{\mathcal{F}}\right\}$ be an SVNS on $\tilde{\mathcal{F}}$. The complement of the set $\alpha_{n}$ (briefly $\alpha_{n}^{c}$ ) defined as follows:

$$
\tilde{\varrho}_{\alpha_{n}^{c}}(v)=\tilde{\zeta} \alpha_{n}(v), \tilde{\varrho}_{\alpha_{n}^{c}}(v)=\left[\tilde{\varrho}_{\alpha_{n}}\right]^{c}(v), \tilde{\zeta}_{\alpha_{n}^{c}}(v)=\tilde{\varrho}_{\alpha_{n}}(v) .
$$


Definition 4 ([9]). Let $\tilde{\mathcal{F}}$ be a non-empty set, $\alpha_{n}, \varepsilon_{n} \in \zeta^{\tilde{\mathcal{F}}}$ be in the form: $\alpha_{n}=\left\{\left\langle v, \tilde{\varrho}_{\alpha_{n}}(v)\right.\right.$, $\left.\left.\tilde{\sigma}_{\alpha_{n}}(v), \tilde{\zeta}_{\alpha_{n}}(v)\right\rangle: v \in \tilde{\mathcal{F}}\right\}$ and $\varepsilon_{n}=\left\{\left\langle v, \tilde{\varrho}_{\varepsilon_{n}}(v), \tilde{\sigma}_{\varepsilon_{n}}(v), \tilde{\zeta}_{\varepsilon_{n}}(v)\right\rangle: v \in \tilde{\mathcal{F}}\right\}$ on $\tilde{\mathcal{F}}$ then,

(a) $\alpha_{n} \subseteq \varepsilon_{n}$ for every $v \in \tilde{\mathcal{F}}$;

$$
\tilde{\varrho}_{\alpha_{n}}(v) \leq \tilde{\varrho}_{\varepsilon_{n}}(v), \tilde{\sigma}_{\alpha_{n}}(v) \geq \tilde{\sigma}_{\mathcal{\varepsilon}_{n}}(v), \tilde{\zeta}_{\alpha_{n}}(v) \geq \tilde{\zeta}_{\varepsilon_{n}}(v) .
$$

(b) $\alpha_{n}=\varepsilon_{n}$ iff $\sigma_{n} \subseteq \varepsilon_{n}$ and $\sigma_{n} \supseteq \varepsilon_{n}$.

(c) $\tilde{0}=\langle 0,1,1\rangle$ and $\tilde{1}=\langle 1,0,0\rangle$.

Definition 5 ([26]). Let $\alpha_{n}, \varepsilon_{n} \in \zeta^{\tilde{\mathcal{F}}}$. Then,

(a) $\alpha_{n} \cap \varepsilon_{n}$ is an SVNS, if for every $v \in \tilde{\mathcal{F}}$,

$$
\alpha_{n} \cap \varepsilon_{n}=\left\langle\left(\tilde{\varrho}_{\alpha_{n}} \cap \tilde{\varrho}_{\varepsilon_{n}}\right)(v),\left(\tilde{\sigma}_{\alpha_{n}} \cup \tilde{\sigma}_{\varepsilon_{n}}\right)(v),\left(\tilde{\zeta}_{\alpha_{n}} \cup \tilde{\zeta}_{\varepsilon_{n}}\right)(v)\right\rangle
$$

where, $\left(\tilde{\varrho}_{\alpha_{n}} \cap \tilde{\varrho}_{\varepsilon_{n}}\right)(v)=\tilde{\varrho}_{\alpha_{n}}(v) \cap 5 \tilde{\varrho}_{\varepsilon_{n}}(v)$ and $\left(\tilde{\zeta}_{\alpha_{n}} \cup \tilde{\zeta}_{\varepsilon_{n}}\right)(v)=\tilde{\zeta}_{\alpha_{n}}(v) \cup \tilde{\zeta}_{\varepsilon_{n}}(v)$, for all $v \in \tilde{\mathcal{F}}$, (b) $\alpha_{n} \cup \varepsilon_{n}$ is an $S V N S$, if for every $v \in \tilde{\mathcal{F}}$,

$$
\alpha_{n} \cup \varepsilon_{n}=\left\langle\left(\tilde{\varrho}_{\alpha_{n}} \cup \tilde{\varrho}_{\varepsilon_{n}}\right)(v),\left(\tilde{\sigma}_{\alpha_{n}} \cap \tilde{\sigma}_{\varepsilon_{n}}\right)(v),\left(\tilde{\zeta}_{\alpha_{n}} \cap \tilde{\zeta}_{\varepsilon_{n}}\right)(v)\right\rangle
$$

Definition 6 ([6]). For any arbitrary family $\left\{\alpha_{n}\right\}_{i \in j} \in \zeta^{\tilde{\mathcal{F}}}$ of SVNS the union and intersection are given by

(a) $\bigcap_{i \in j}\left[\alpha_{n}\right]_{i}=\left\langle\cap_{i \in j} \tilde{Q}_{\left[\alpha_{n}\right]_{i}}(v), \cup_{i \in j} \tilde{\sigma}_{\left[\alpha_{n}\right]_{i}}(v), \cup_{i \in j} \tilde{S}_{\left[\alpha_{n}\right]_{i}}(v)\right\rangle$,

(b) $\bigcup_{i \in j}\left[\alpha_{n}\right]_{i}=\left\langle\cup_{i \in j} \tilde{\varrho}_{\left[\alpha_{n}\right]_{i}}(v), \cap_{i \in j} \tilde{\sigma}_{\left[\alpha_{n}\right]_{i}}(v), \cap_{i \in j} \tilde{S}_{\left[\alpha_{n}\right]_{i}}(v)\right\rangle$.

Definition 7 ([18]). A single-valued neutrosophic topological spaces is an ordered $\left(\tilde{\mathcal{F}}, \tilde{\tau}^{\tilde{\varphi}}, \tilde{\tau}^{\tilde{\sigma}}, \tilde{\tau}^{\tilde{\tau}}\right)$ where $\tilde{\tau}^{\tilde{\rho}}, \tilde{\tau}^{\tilde{\sigma}}, \tilde{\tau}^{\tilde{s}}: \zeta^{\tilde{\mathcal{F}}} \rightarrow \zeta$ is a mapping satisfying the following axioms:

$(S V N T 1) \quad \tilde{\tau}^{\tilde{\rho}}(\tilde{0})=\tilde{\tau}^{\tilde{\rho}}(\tilde{1})=\tilde{\tau}^{\tilde{\sigma}}(\tilde{0})=\tilde{\tau}^{\tilde{\sigma}}(\tilde{1})=0$ and $\tilde{\tau}^{\tilde{s}}(\tilde{0})=\tilde{\tau}^{\tilde{s}}(\tilde{1})=1$.

(SVNT2) $\quad \tilde{\tau}^{\tilde{\rho}}\left(\alpha_{n} \cap \varepsilon_{n}\right) \geq \tilde{\tau}^{\tilde{\rho}}\left(\alpha_{n}\right) \cap \tilde{\tau}^{\tilde{\rho}}\left(\varepsilon_{n}\right), \quad \tilde{\tau}^{\tilde{\sigma}}\left(\alpha_{n} \cap \varepsilon_{n}\right) \leq \tau^{\tilde{\sigma}}\left(\alpha_{n}\right) \cup \tilde{\tau}^{\tilde{\sigma}}\left(\varepsilon_{n}\right)$, $\tilde{\tau}^{\tilde{s}}\left(\alpha_{n} \cap \varepsilon_{n}\right) \leq \tilde{\tau}^{\tilde{s}}\left(\alpha_{n}\right) \cup \tilde{\tau}^{\tilde{s}}\left(\varepsilon_{n}\right)$, for every $\alpha_{n}, \varepsilon_{n} \in \zeta^{\tilde{\mathcal{F}}}$,

(SVNT3) $\quad \tilde{\tau} \tilde{\varrho}\left(\cup_{j \in \Gamma}\left[\alpha_{n}\right]_{j}\right) \geq \cap_{j \in \Gamma} \tilde{\tau} \tilde{\varrho}\left(\left[\alpha_{n}\right]_{j}\right), \quad \tilde{\tau} \tilde{\sigma}\left(\cup_{i \in \Gamma}\left[\alpha_{n}\right]_{j}\right) \leq \cup_{j \in \Gamma} \tilde{\tau}^{\tilde{\sigma}}\left(\left[\alpha_{n}\right]_{j}\right)$, $\tilde{\tau} \tilde{s}\left(\cup_{j \in \Gamma}\left[\alpha_{n}\right]_{j}\right) \leq \cup_{j \in \Gamma} \tilde{\tau} \tilde{s}\left(\left[\alpha_{n}\right]_{j}\right)$, for every $\left[\alpha_{n}\right]_{j} \in \zeta^{\tilde{\mathcal{F}}}$.

The quadruple $\left(\tilde{\mathcal{F}}, \tilde{\tau}^{\tilde{\varrho}}, \tilde{\tau}^{\tilde{\sigma}}, \tilde{\tau}^{\tilde{s}}\right)$ is called a single-valued neutrosophic topological space (briefly, SVNT, for short). Occasionally we write $\tau^{\tilde{\rho} \tilde{\sigma} \tilde{s}}$ for $\left(\tilde{\tau}^{\tilde{\varrho}}, \tilde{\tau}^{\tilde{\sigma}}, \tilde{\tau}^{\tilde{s}}\right)$ and it will cause no ambiguity.

Definition 8 ([21]). Let $\left(\tilde{\mathcal{F}}, \tau^{\tilde{\sigma} \tilde{\sigma} \tilde{S}}\right)$ be an SVNTS . Then, for every $\alpha_{n} \in \zeta^{\tilde{\mathcal{F}}}$ and $r \in \zeta_{0}$. Then the single-valued neutrosophic closure and single-valued neutrosophic interior of $\alpha_{n}$ are defined by:

$$
\begin{gathered}
\mathrm{C}_{\tau \tilde{\tilde{\rho} \tilde{\sigma}} \tilde{\tilde{S}}}\left(\alpha_{n}, r\right)=\bigcap\left\{\varepsilon_{n} \in \zeta^{\tilde{\mathcal{F}}}: \alpha_{n} \leq \varepsilon_{n}, \tau^{\tilde{\varrho}}\left(\left[\varepsilon_{n}\right]^{c}\right) \geq r, \tau^{\tilde{\sigma}}\left(\left[\varepsilon_{n}\right]^{c}\right) \leq 1-r, \tau^{\tilde{\varsigma}}\left(\left[\varepsilon_{n}\right]^{c}\right) \leq 1-r\right\}, \\
\operatorname{int}_{\tau} \tau_{\tilde{\rho} \tilde{\sigma} \tilde{\zeta}}\left(\alpha_{n}, r\right)=\bigcup\left\{\varepsilon_{n} \in \zeta^{\tilde{\mathcal{F}}}: \alpha_{n} \geq \varepsilon_{n}, \tau^{\tilde{\varrho}}\left(\varepsilon_{n}\right) \geq r, \tau^{\tilde{\sigma}}\left(\varepsilon_{n}\right) \leq 1-r, \tau^{\tilde{\zeta}}\left(\varepsilon_{n}\right) \leq 1-r\right\} .
\end{gathered}
$$

Definition 9 ([24,25]). Let $\left(\tilde{\mathcal{F}}, \tau^{\tilde{\varrho} \tilde{\sigma} \tilde{S}}\right)$ be an SVNTS and $\alpha_{n} \in \zeta^{\tilde{\mathcal{F}}}, r \in \zeta_{0}$. Then,

(1) $\alpha_{n}$ is said to be $r$-single-valued neutrosophic semi-open (briefly, $r$-SVNSO) iff

$$
\alpha_{n} \leq \mathrm{C}_{\tilde{\tau} \tilde{\widetilde{\sigma}} \tilde{\sigma} \tilde{\zeta}}\left(\operatorname{int}_{\tilde{\tau} \tilde{\rho} \tilde{\sigma} \tilde{G}}\left(\left[\alpha_{n}\right]_{r}, r\right), r\right),
$$

(2) $\alpha_{n}$ is said to be $r$-single-valued neutrosophic $\beta$-open (briefly, $r$-SVN $\beta O$ ) iff

$$
\alpha_{n} \leq \mathrm{C}_{\tilde{\tau} \tilde{\rho} \tilde{\sigma} \tilde{\zeta}}\left(\operatorname{int}_{\tilde{\tau} \tilde{\widetilde{\sigma}} \tilde{\sigma}}\left(\mathrm{C}_{\tilde{\tau} \tilde{\sigma} \tilde{\sigma} \tilde{\zeta}}\left(\left[\alpha_{n}\right]_{r}, r\right), r\right), r\right) .
$$


(3) $\alpha_{n}$ is said to be $r$-single-valued neutrosophic regular open (briefly, $r$-SVNRO) iff

$$
\alpha_{n}=\operatorname{int}_{\tilde{\tau} \tilde{\tilde{\rho}} \tilde{\tilde{S}} \tilde{\tilde{s}}}\left(\mathrm{C}_{\tilde{\tau} \tilde{\rho} \tilde{\tilde{\rho}} \tilde{\tilde{S}}}\left(\left[\alpha_{n}\right]_{r}, r\right), r\right),
$$

The complement of r-SVNSO (resp, r-SVN $\beta O$ ) are said to be r-SVNSC (resp, r-SVN $\beta C)$ ), respectively.

Definition 10 ([21]). Let $(\tilde{\mathcal{F}})$ be a non-empty set and $v \in \tilde{\mathcal{F}}$, let $s \in(0,1], t \in[0,1)$ and $k \in[0,1)$, then the single-valued neutrosophic point $x_{s, t, k}$ in $\tilde{\mathcal{F}}$ given by

$$
x_{s, t, k}(v)= \begin{cases}(s, t, k), & \text { if } x=v, \\ (0,1,1), & \text { otherwise. }\end{cases}
$$

We say that, $x_{s, t, p} \in \alpha_{n}$ iff $s<\tilde{\varrho}_{\alpha_{n}}(v), t \geq \tilde{\sigma}_{\alpha_{n}}(v)$ and $k \geq \tilde{\tilde{\zeta}}_{\alpha_{n}}(v)$. We indicate the set of all single-valued neutrosophic points in $\tilde{\mathcal{F}}$ as $\mathrm{P}_{x_{s, t}, k}(\tilde{\mathcal{F}})$. A single-valued neutrosophic set $\alpha_{n}$ is said to be quasi-coincident with another single-valued neutrosophic set $\varepsilon_{n}$, denoted by $\alpha_{n} q \varepsilon_{n}$, if there exists an element $v \in \tilde{\mathcal{F}}$ such that

$$
\tilde{\varrho}_{\alpha_{n}}(v)+\tilde{\varrho}_{\varepsilon_{n}}(v)>1, \quad \tilde{\sigma}_{\alpha_{n}}(v)+\tilde{\sigma}_{\mathcal{E}_{n}}(v) \leq 1, \quad \tilde{\zeta}_{\alpha_{n}}(v)+\tilde{\zeta}_{\varepsilon_{n}}(v) \leq 1 .
$$

Definition 11 ([21]). A mapping $£^{\tilde{Q}}, £^{\tilde{\sigma}}, £^{\tilde{S}}: \zeta^{\tilde{\mathcal{F}}} \rightarrow \zeta$ is called single-valued neutrosophic ideal (SVNI) on $\tilde{\mathcal{F}}$ if it satisfies the following conditions:

$\left(£_{1}\right) \quad £^{\tilde{Q}}(\tilde{0})=1$ and $£^{\tilde{\sigma}}(\tilde{0})=£^{\tilde{\zeta}}(\tilde{0})=0$.

$\left(£_{2}\right) \quad$ If $\sigma_{n} \leq \gamma_{n}$, then $£^{\tilde{Q}}\left(\varepsilon_{n}\right) \leq £^{\tilde{Q}}\left(\alpha_{n}\right), \quad £^{\tilde{\sigma}}\left(\varepsilon_{n}\right) \geq £^{\tilde{\sigma}}\left(\alpha_{n}\right)$ and $£^{\tilde{S}}\left(\varepsilon_{n}\right) \geq £^{\tilde{S}}\left(\alpha_{n}\right)$, for every $\varepsilon_{n}, \alpha_{n} \in \zeta^{\tilde{\mathcal{F}}}$.

$\left(£_{3}\right) \quad £^{\tilde{\varrho}}\left(\alpha_{n} \cup \varepsilon_{n}\right) \geq £^{\tilde{\varrho}}\left(\alpha_{n}\right) \cap £^{\tilde{\varrho}}\left(\varepsilon_{n}\right), \quad £^{\tilde{\sigma}}\left(\alpha_{n} \cup \varepsilon_{n}\right) \leq £^{\tilde{\sigma}}\left(\alpha_{n}\right) \cup £^{\tilde{\sigma}}\left(\varepsilon_{n}\right)$ and $£^{\tilde{S}}\left(\alpha_{n} \cup \varepsilon_{n}\right) \leq £^{\tilde{S}}\left(\alpha_{n}\right) \cup £^{\tilde{S}}\left(\varepsilon_{n}\right)$, for every $\alpha_{n}, \varepsilon_{n} \in \zeta^{\tilde{\mathcal{F}}}$.

The triable $\left(\tilde{\mathcal{F}}, \tau^{\tilde{\rho} \tilde{\sigma} \tilde{S}}, £^{\tilde{\rho} \tilde{\sigma} \tilde{S}}\right)$ is called a single-valued neutrosophic ideal topological space in the Šostak sense (briefly, SVNITS).

Definition 12 ([21]). Let $\left(\tilde{\mathcal{F}}, \tau^{\tilde{\rho} \tilde{\sigma} \tilde{S}}, £^{\tilde{\rho} \tilde{\sigma} \tilde{S}}\right)$ be an SVNITS for each $\alpha_{n} \in \zeta^{\tilde{\mathcal{F}}}$. Then, the single-valued neutrosophic ideal open local function $\left[\alpha_{n}\right]_{r}^{\odot}\left(\tau^{\tilde{\rho} \tilde{\sigma} \tilde{S}}, £^{\tilde{\sigma} \tilde{\sigma} \tilde{S}}\right)$ of $\alpha_{n}$ is the union of all single-valued neutrosophic points $x_{s, t, k}$ such that if $\varepsilon_{n} \in Q_{\tau \tilde{\tilde{\sigma}} \tilde{\tilde{\sigma}}}\left(x_{s, t, k}, r\right)$ and $£^{\tilde{\varrho}}\left(\omega_{n}\right) \geq r, £^{\tilde{\sigma}}\left(\omega_{n}\right) \leq 1-r$, $£^{\tilde{S}}\left(\omega_{n}\right) \leq 1-r$, then there is at least one $v \in \tilde{\mathcal{F}}$ for which

$$
\tilde{\varrho}_{\alpha_{n}}(v)+\tilde{\varrho}_{\varepsilon_{n}}(v)-1>\tilde{\varrho}_{\omega_{n}}(v), \quad \tilde{\sigma}_{\alpha_{n}}(v)+\tilde{\sigma}_{\varepsilon_{n}}(v)-1 \leq \tilde{\sigma}_{\omega_{n}}(v), \quad \tilde{\zeta}_{\alpha_{n}}(v)+\tilde{\zeta}_{\varepsilon_{n}}(v)-1 \leq \tilde{\zeta} \omega_{n}(v)
$$

Occasionally, we will write $\left[\alpha_{n}\right]_{r}^{\odot}$ for $\left[\alpha_{n}\right]_{r}^{\odot}\left(\tau^{\tilde{\varrho} \tilde{\sigma} \tilde{\zeta}}, £^{\tilde{\varrho} \tilde{\sigma} \tilde{S}}\right)$ and it will have no ambiguity.

$\operatorname{Remark} 1$ ([21]). Let $\left(\tilde{\mathcal{F}}, \tau^{\tilde{Q} \tilde{\sigma} \tilde{S}}, £^{\tilde{Q} \tilde{\sigma} \tilde{S}}\right)$ be an SVNITS and $\alpha_{n} \in \zeta^{\tilde{\mathcal{F}}}$, we can define

$$
\mathrm{CI}_{\tau \tilde{\tau} \tilde{\sigma} \tilde{\zeta}}^{\odot}\left(\alpha_{n}, r\right)=\alpha_{n} \cup\left[\alpha_{n}\right]_{r}^{\odot}, \quad \operatorname{int}_{\tau^{\tilde{\sigma} \tilde{\sigma} \tilde{\zeta}}}^{\odot}\left(\alpha_{n}, r\right)=\alpha_{n} \cap\left[\left(\alpha_{n}^{c}\right)_{r}^{\odot}\right]^{c} .
$$

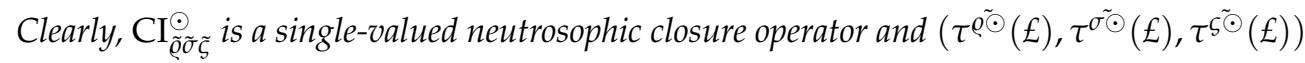
is the single-valued neutrosophic topology generated by $\mathrm{CI}_{\tau^{\tilde{\rho} \tilde{\sigma} \tilde{\zeta}}}^{\odot}$, i.e.,

$$
\tau^{\odot}(\mathcal{I})\left(\alpha_{n}\right)=\bigcup\left\{r \mid \mathrm{CI}_{\tilde{\tau} \tilde{\tilde{\sigma} \tilde{\sigma} \tilde{\zeta}}}^{\odot}\left(\alpha_{n}^{c}, r\right)=\alpha_{n}^{c}\right\} .
$$

Definition 13 (25). An SVNS $\delta$ in $\delta: \tilde{\mathcal{F}} \times \tilde{\mathcal{F}}$ is called a single-valued neutrosophic relation $(S V N R)$ in $\tilde{\mathcal{F}}$, denoted by $\delta=\left\{\left\langle(\omega, v), \tilde{\varrho}_{\delta}(\omega, v), \tilde{\sigma}_{\delta}(\omega, v), \tilde{\varsigma}_{\delta}(\omega, v)\right\rangle \mid(\omega, v) \in \tilde{\mathcal{F}} \times \tilde{\mathcal{F}}\right\}$, where $\tilde{\varrho}_{\delta}: \tilde{\mathcal{F}} \times \tilde{\mathcal{F}} \Rightarrow[0,1], \tilde{\sigma}_{\delta}: \tilde{\mathcal{F}} \times \tilde{\mathcal{F}} \Rightarrow[0,1]$ and $\tilde{\zeta}_{\delta}: \tilde{\mathcal{F}} \times \tilde{\mathcal{F}} \Rightarrow[0,1]$ denote the truth-membership function, indeterminacy membership function and falsity-membership function of $\delta$, respectively. In what follows, $\operatorname{SVNR}(\tilde{\mathcal{F}})$ will denote the family of all single-valued neutrosophic relations in $\tilde{\mathcal{F}}$. 


\section{Single-Valued Neutrosophic Semi-Closure Spaces in Šostak Sense}

We begin this section by defining the notion of single-valued neutrosophic semiclosure space. Some of its characteristic properties are considered. Further, we present and explore the properties and characterizations of the single-valued neutrosophic operators, namely $\beta £$-closure $\left(\beta £ C_{\tau \tilde{\sigma} \tilde{\sigma} \tilde{\zeta}}\right)$ and $\beta £$-interior $\left(\beta £\right.$ int $\left._{\tau} \tilde{\tau \tilde{\sigma} \tilde{\zeta}}\right)$ in the single-valued neutrosophic ideal topological space $\left(\tilde{\mathcal{F}}, \tau^{\tilde{O} \tilde{\sigma} \tilde{S}}, £^{\tilde{O} \tilde{\sigma} \tilde{S}}\right)$.

Definition 14. A mapping $\mathbb{S C}: \zeta^{\tilde{\mathcal{F}}} \times \zeta_{0} \rightarrow \zeta^{\mathcal{F}}$ is called a single-valued neutrosophic semi-closure operator on $\mathcal{F}$ if, for every $\alpha_{n}, \varepsilon_{n} \in \zeta^{\mathcal{F}}$ and $r, s \in \zeta_{0}$, the following axioms are satisfied:

$\left.\left(\mathbb{S C}_{1}\right) \mathbb{S C}(\tilde{0}), r\right)=\tilde{0}$

$\left(\mathbb{S C}_{2}\right) \alpha_{n} \leq \mathbb{S C}\left(\alpha_{n}, r\right)$

$\left(\mathbb{S C}_{3}\right) \mathbb{S C}\left(\alpha_{n}, r\right) \vee \mathbb{S C}\left(\varepsilon_{n}, r\right)=\mathbb{S C}\left(\alpha_{n} \vee \varepsilon_{n}, r\right)$,

$\left(\mathbb{S C}_{4}\right) \mathbb{S C}\left(\alpha_{n}, s\right) \leq \mathbb{S C}\left(\alpha_{n}, r\right)$ if $s \leq r$

$\left(\mathbb{S C}_{5}\right) \mathbb{S C}\left(\mathbb{S} \mathbb{C}\left(\alpha_{n}, s\right), r\right)=\mathbb{S} \mathbb{C}\left(\alpha_{n}, r\right)$.

The pair $(X, \mathbb{S C})$ is a single-valued neutrosophic semi-closure space $(\mathcal{S V N S S C S}$, for short).

If $\mathbb{S C}_{1}$ and $\mathbb{S C}_{2}$ are single-valued neutrosophic closure operators on $\mathcal{F}$. Then, $\mathbb{S C}_{1}$ is finer than $\mathbb{C}_{2}$, denoted by $\mathbb{S C}_{2} \leq \mathbb{S C}_{1}$ iff $\mathbb{S C}_{1}\left(\alpha_{n}, r\right) \leq \mathbb{S C}_{2}\left(\alpha_{n}, r\right)$, for every $\alpha_{n} \in \zeta^{\tilde{\mathcal{F}}}$ and $r \in \zeta_{0}$.

Theorem 1. Let $\left(\tilde{\mathcal{F}}, \tau^{\tilde{\varrho} \tilde{\sigma} \tilde{S}}\right)$ be an SVNTS. Then, for any $\alpha_{n} \in \zeta^{\tilde{\mathcal{F}}}$ and $r \in \zeta_{0}$, we define an operator $\mathbb{S C}_{\tau} \tau_{\tilde{\sigma} \tilde{\sigma} \tilde{\xi}}: \zeta^{\tilde{\mathcal{F}}} \times \zeta_{0} \rightarrow \zeta^{\tilde{\mathcal{F}}}$ as follows:

$$
\mathbb{S C}_{\tau} \tilde{\tilde{\rho} \tilde{\tilde{\sigma}} \tilde{\tilde{C}}}\left(\alpha_{n}, s\right)=\bigwedge\left\{\varepsilon_{n} \in \zeta^{\tilde{\mathcal{F}}}: \alpha_{n} \leq \varepsilon_{n}, \quad \varepsilon_{n} \text { is r-SVNSC }\right\} .
$$

Then, $\left(\mathcal{F}, \mathbb{S C}_{\tau} \tilde{\rho} \tilde{\sigma} \tilde{\sigma}\right)$ is an $\mathcal{S} \mathcal{V N} \mathcal{S C S}$.

Proof. Suppose that $\left(\tilde{\mathcal{F}}, \tau^{\tilde{\varrho} \tilde{\sigma} \tilde{S}}\right)$ is an SVNTS. Then, $\left(\mathbb{S C}_{1}\right),\left(\mathbb{S C}_{2}\right)$ and $\left(\mathbb{S C}_{4}\right)$ follows directly from the definition of $\mathbb{S} \mathbb{C}_{\tau} \tilde{\tilde{\sigma} \tilde{\sigma} \tilde{s}}$.

$\left(\mathbb{S C}_{3}\right)$ Since $\alpha_{n}, \varepsilon_{n} \leq \alpha_{n} \cup \varepsilon_{n}$ we obtain $\mathbb{S C}_{\tau} \tilde{\rho}^{\tilde{\sigma} \tilde{\sigma} \tilde{\zeta}}\left(\varepsilon_{n}, r\right) \leq \mathbb{S C}_{\tau} \tilde{\tilde{\sigma} \tilde{\sigma} \tilde{\xi}}\left(\alpha_{n} \cup \varepsilon_{n}, r\right)$ and $\mathbb{S C}_{\tau}^{\tilde{\sigma} \tilde{\sigma} \tilde{\sigma}}$ $\left(\alpha_{n}, r\right) \leq \mathbb{S C}_{\tau} \tilde{\rho} \tilde{\sigma} \tilde{s}\left(\alpha_{n} \cup \varepsilon_{n}, r\right)$, therefore,

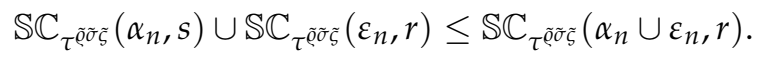

Let $\left(\tilde{\mathcal{F}}, \tau^{\tilde{\rho} \tilde{\sigma} \tilde{S}}\right)$ be an SVNTS. From $\left(\mathbb{S C}_{2}\right)$, we have

$$
\begin{aligned}
& \alpha_{n} \leq \mathbb{S C}_{\tau \tilde{\rho}}\left(\alpha_{n}, r\right), \quad\left[\mathbb{S C}_{\tau \tilde{\rho}}\left(\alpha_{n}, r\right)\right]^{c} \leq \mathbb{C}_{\tau \tilde{\rho}}\left(\operatorname{int}_{\tau^{\tilde{\rho}}}\left(\left[\operatorname{SC}_{\tau^{\tilde{\rho}}}\left(\alpha_{n}, r\right)\right]^{c}, r\right), r\right) \\
& {\left[\mathbb{S C}_{\tau_{\tilde{\sigma}}}\left(\alpha_{n}, r\right)\right]^{c} \geq \mathbb{C}_{\tau \tilde{\sigma}}\left(\operatorname{int}_{\tau^{\tilde{\sigma}}}\left(\left[\operatorname{SC}_{\tau^{\tilde{\sigma}}}\left(\alpha_{n}, r\right)\right]^{c}, r\right), r\right),} \\
& {\left[\mathbb{S C}_{\tau \tilde{\zeta}}\left(\alpha_{n}, r\right)\right]^{c} \geq \mathbb{C}_{\tau \tilde{\zeta}}\left(\operatorname{int}_{\tau \tilde{\zeta}}\left(\left[\mathbb{S C}_{\tau \tilde{\zeta}}\left(\alpha_{n}, r\right)\right]^{c}, r\right), r\right)} \\
& \varepsilon_{n} \leq \mathbb{S C}_{\tau^{\tilde{\rho}}}\left(\varepsilon_{n}, r\right), \quad\left[\mathbb{S C}_{\tau^{\tilde{\rho}}}\left(\varepsilon_{n}, r\right)\right]^{c} \leq \mathrm{C}_{\tau^{\tilde{\rho}}}\left(\operatorname{int}_{\tau^{\tilde{\rho}}}\left(\left[\mathbb{S C}_{\tau^{\tilde{\rho}}}\left(\varepsilon_{n}, r\right)\right]^{c}, r\right), r\right) \\
& {\left[\mathbb{S C}_{\tau^{\tilde{\sigma}}}\left(\varepsilon_{n}, r\right)\right]^{c} \geq \mathbb{C}_{\tau^{\tilde{\sigma}}}\left(\operatorname{int}_{\tau^{\tilde{\sigma}}}\left(\left[\operatorname{SC}_{\tau^{\tilde{\sigma}}}\left(\varepsilon_{n}, r\right)\right]^{c}, r\right), r\right),} \\
& {\left[\mathbb{S C}_{\tau \tilde{\xi}}\left(\varepsilon_{n}, r\right)\right]^{c} \geq \mathbb{C}_{\tau \tilde{\xi}}\left(\operatorname{int}_{\tau \tilde{\xi}}\left(\left[\mathbb{S C}_{\tau \tilde{\xi}}\left(\varepsilon_{n}, r\right)\right]^{c}, r\right), r\right)}
\end{aligned}
$$

It implies that $\alpha_{n} \cup \varepsilon_{n} \leq \mathbb{S C}_{\tau} \tilde{\tilde{\rho} \tilde{\sigma} \tilde{\zeta}}\left(\alpha_{n}, r\right) \cup \mathbb{S C}_{\tau} \tilde{\tilde{\sigma} \tilde{\sigma} \tilde{\zeta}}\left(\varepsilon_{n}, r\right)$ and

$$
\begin{aligned}
& {\left[\mathbb{S C}_{\tau \tilde{\varrho}}\left(\alpha_{n}, r\right) \cup \mathbb{S C}_{\tau \tilde{\rho}}\left(\varepsilon_{n}, r\right)\right]^{c}=\left[\mathbb{S C}_{\tau \tilde{\rho}}\left(\alpha_{n}\right]^{c} \cap\left[\mathbb{S C}_{\tau \tilde{\rho}}\left(\varepsilon_{n}, r\right)\right]^{c}\right.}
\end{aligned}
$$

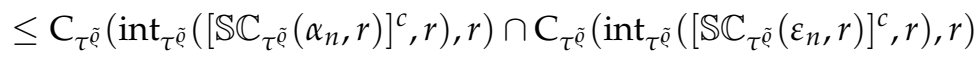

$$
\begin{aligned}
& =\mathrm{C}_{\tau \tilde{\rho}}\left(\operatorname{int}_{\tau^{\tilde{\rho}}}\left(\left[\mathbb{S C}_{\tau^{\tilde{\rho}}}\left(\alpha_{n}, r\right)\right]^{c} \cap\left[\mathbb{S C}_{\tau^{\tilde{\rho}}}\left(\varepsilon_{n}, r\right)\right]^{c}, r\right), r\right) \\
& =\mathrm{C}_{\tau^{\tilde{\rho}}}\left(\operatorname{int}_{\tau^{\tilde{\rho}}}\left(\left[\mathbb{S C}_{\tau^{\tilde{\rho}}}\left(\alpha_{n}, r\right) \cup \mathbb{S C}_{\tau^{\tilde{\rho}}}\left(\varepsilon_{n}, r\right)\right]^{c}, r\right), r\right) \text {, }
\end{aligned}
$$




$$
\begin{aligned}
& {\left[\mathbb{S C}_{\tau^{\tilde{\sigma}}}\left(\alpha_{n}, r\right) \cup \mathbb{S C}_{\tau^{\tilde{\sigma}}}\left(\varepsilon_{n}, r\right)\right]^{c}=\left[\mathbb{S C}_{\tau^{\tilde{\sigma}}}\left(\alpha_{n}\right]^{c} \cap\left[\mathbb{S C}_{\tau_{\tilde{\sigma}}}\left(\varepsilon_{n}, r\right)\right]^{c}\right.} \\
& \geq \mathrm{C}_{\tau^{\tilde{\sigma}}}\left(\operatorname{int}_{\tau^{\tilde{\sigma}}}\left(\left[\mathbb{S C}_{\tau^{\tilde{\sigma}}}\left(\alpha_{n}, r\right)\right]^{c}, r\right), r\right) \cap \mathrm{C}_{\tau^{\tilde{\sigma}}}\left(\operatorname{int}_{\tau^{\tilde{\sigma}}}\left(\left[\mathbb{S C}_{\tau^{\tilde{\sigma}}}\left(\varepsilon_{n}, r\right)\right]^{c}, r\right), r\right) \\
& =\mathrm{C}_{\tau^{\tilde{\sigma}}}\left(\operatorname{int}_{\tau^{\tilde{\sigma}}}\left(\left[\mathbb{S C}_{\tau^{\tilde{\sigma}}}\left(\alpha_{n}, r\right)\right]^{c} \cap\left[\mathbb{S C}_{\tau^{\tilde{\sigma}}}\left(\varepsilon_{n}, r\right)\right]^{c}, r\right), r\right) \\
& =\mathrm{C}_{\tau^{\tilde{\sigma}}}\left(\operatorname{int}_{\tau^{\tilde{\sigma}}}\left(\left[\mathbb{S C}_{\tau^{\tilde{\sigma}}}\left(\alpha_{n}, r\right) \cup \mathbb{S C}_{\tau^{\tilde{\sigma}}}\left(\varepsilon_{n}, r\right)\right]^{c}, r\right), r\right) \\
& {\left[\mathbb{S C}_{\tau^{\tilde{\zeta}}}\left(\alpha_{n}, r\right) \cup \mathbb{S C}_{\tau^{\tilde{\zeta}}}\left(\varepsilon_{n}, r\right)\right]^{c}=\left[\mathbb{S C}_{\tau \tilde{\zeta}}\left(\alpha_{n}\right]^{c} \cap\left[\mathbb{S C}_{\tau^{\tilde{\zeta}}}\left(\varepsilon_{n}, r\right)\right]^{c}\right.} \\
& \geq \mathrm{C}_{\tau^{\tilde{\xi}}}\left(\operatorname{int}_{\tau \tilde{\zeta}}\left(\left[\mathbb{S C}_{\tau^{\tilde{\zeta}}}\left(\alpha_{n}, r\right)\right]^{c}, r\right), r\right) \cap \mathrm{C}_{\tau \tilde{\zeta}}\left(\operatorname{int}_{\tau \tilde{\zeta}}\left(\left[\mathbb{S C}_{\tau^{\tilde{\zeta}}}\left(\varepsilon_{n}, r\right)\right]^{c}, r\right), r\right) \\
& =\mathrm{C}_{\tau \tilde{\zeta}}\left(\operatorname{int}_{\tau \tilde{\tau}}\left(\left[\mathbb{S C}_{\tau \tilde{\zeta}}\left(\alpha_{n}, r\right)\right]^{c} \cap\left[\mathbb{S C}_{\tau \tilde{\zeta}}\left(\varepsilon_{n}, r\right)\right]^{c}, r\right), r\right) \\
& =\mathrm{C}_{\tau \tilde{\xi}}\left(\operatorname{int}_{\tau \tilde{\xi}}\left(\left[\mathbb{S C}_{\tau^{\tilde{\xi}}}\left(\alpha_{n}, r\right) \cup \mathbb{S C}_{\tau^{\tilde{\sigma}}}\left(\varepsilon_{n}, r\right)\right]^{c}, r\right), r\right)
\end{aligned}
$$

Hence, $\mathbb{S C}_{\tau} \tilde{\tilde{\sigma} \tilde{\sigma} \tilde{\xi}}\left(\alpha_{n}, s\right) \cup \mathbb{S C}_{\tau} \tilde{\tilde{\sigma} \tilde{\sigma} \tilde{\xi}}\left(\varepsilon_{n}, r\right) \geq \mathbb{S C}_{\tau} \tilde{\tilde{\rho} \tilde{\sigma} \tilde{\xi}}\left(\alpha_{n} \cup \varepsilon_{n}, r\right)$; therefore,

$$
\mathbb{S C}_{\tau \tilde{\rho} \tilde{\sigma} \tilde{\xi}}\left(\alpha_{n}, s\right) \cup \mathbb{S C}_{\tau} \tau_{\tilde{\rho} \tilde{\sigma} \tilde{\xi}}\left(\varepsilon_{n}, r\right)=\mathbb{S C}_{\tau \tilde{\sigma} \tilde{\sigma} \tilde{\xi}}\left(\alpha_{n} \cup \varepsilon_{n}, r\right) .
$$

$\left(\mathbb{S C}_{5}\right)$ Suppose that there exists $r \in \zeta_{0}, \alpha_{n} \in \zeta^{\mathcal{F}}$ and $\kappa \in \mathcal{F}$ such that

$$
\begin{aligned}
& \tau_{\mathbb{S}_{\tau^{\tilde{\rho}}}^{\tilde{\tilde{\rho}}}}^{\tilde{\tilde{S}}} \mathbb{S}_{\left.\tau_{\tilde{\rho}}^{\tilde{\rho}}\left(\alpha_{n}, r\right), r\right)}(v)>\tau_{\mathbb{S C}_{\tau^{\tilde{\rho}}}^{\tilde{\tilde{\rho}}}\left(\alpha_{n}, r\right)}(v) . \\
& \tau_{\mathbb{S} \mathbb{C}_{\tau^{\tilde{\sigma}}}\left(\mathbb{S C}_{\tau^{\tilde{\sigma}}}\left(\alpha_{n}, r\right), r\right)}(v) \leq \tau_{\mathbb{S C}_{\tau^{\tilde{\sigma}}}\left(\alpha_{n}, r\right)}(v) \text {. }
\end{aligned}
$$

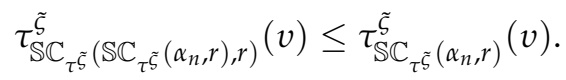

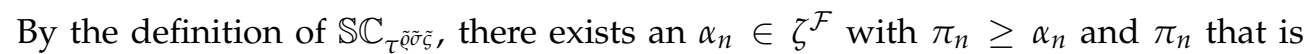
$r$-SVNSC such that

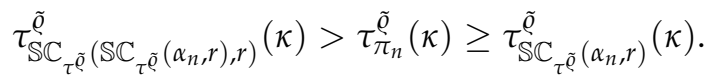

$$
\begin{aligned}
& \tau_{\mathbb{S C}_{\tau^{\tilde{\sigma}}}\left(\mathbb{S C}_{\tau^{\tilde{\sigma}}}\left(\alpha_{n}, r\right), r\right)}(\kappa) \leq \tau_{\pi_{n}}^{\tilde{\sigma}}(\kappa)<\tau_{\mathbb{S}_{\tau^{\tilde{\sigma}}}}^{\tilde{\sigma}}\left(\alpha_{n}, r\right)(\kappa) \text {. }
\end{aligned}
$$

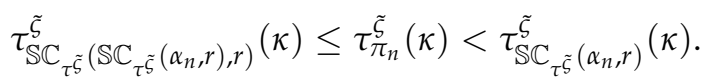

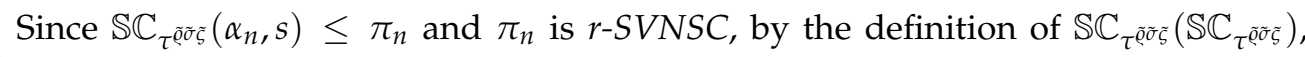
we have

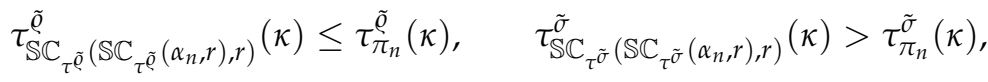

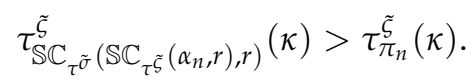

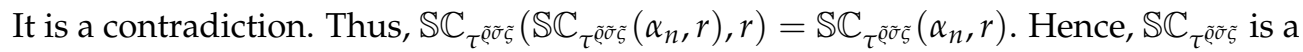
single-valued neutrosophic semi-closure operator on $\mathcal{F}$.

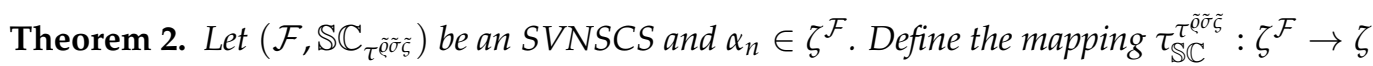
on $\mathcal{F}$ by

$$
\begin{gathered}
\tau_{\mathbb{S} \mathbb{C}}^{\tilde{\rho}}\left(\alpha_{n}\right)=\bigcup\left\{r \in \zeta_{0} \mid \mathbb{S C}_{\tau^{e}}\left(\left[\alpha_{n}\right]^{c}, r\right)=\left[\alpha_{n}\right]^{c}\right\}, \\
\tau_{\mathbb{S} \mathbb{C}}^{\tilde{\sigma}}\left(\alpha_{n}\right)=\bigcap\left\{1-r \in \zeta_{0} \mid \mathbb{S C}_{\tau^{\sigma}}\left(\left[\alpha_{n}\right]^{c}, r\right)=\left[\alpha_{n}\right]^{c}\right\}, \\
\tau_{\mathbb{S} \mathbb{C}}^{\tilde{\varsigma}}\left(\alpha_{n}\right)=\bigcap\left\{1-r \in \zeta_{0} \mid \mathbb{S C}_{\tau^{c}}\left(\left[\alpha_{n}\right]^{c}, r\right)=\left[\alpha_{n}\right]^{c}\right\},
\end{gathered}
$$

Then,

(1) $\tau_{\mathbb{S C}}^{\tau \tilde{C} \tilde{C} \tilde{F}}$ is an SVNTS on $\mathcal{F}$;

(2) $\mathbb{S C}_{\mathbb{S C}}^{\tau \tilde{Q} \tilde{\sigma} \tilde{S}}$ is finer than $\mathbb{S C}$. 
Proof. (SVNT1) Let $\left(\mathcal{F}, \mathbb{S C}_{\tau} \tilde{\tau} \tilde{\sigma} \tilde{\xi}\right)$ be an $S V N S C S$. Since $\mathbb{S C}(\tilde{0}, r)=\tilde{0}$ and $\mathbb{S} \mathbb{C}(\tilde{1}, r)=\tilde{1}$ for every $r \in \zeta_{0}$,

$(\mathrm{SVNT2})$ Let $\left(\mathcal{F}, \mathbb{S C}_{\tau} \tilde{\tilde{\sigma} \tilde{\sigma} \tilde{\zeta}}\right)$ be an SVNSCS. Suppose that there exists $\left[\alpha_{n}\right]_{1},\left[\alpha_{n}\right]_{2} \in \zeta^{\mathcal{F}}$ such that

$$
\begin{array}{r}
\tau_{\mathbb{S} \mathbb{C}}^{\tilde{\sigma}}\left(\left[\alpha_{n}\right]_{1} \cap\left[\alpha_{n}\right]_{2}\right)<\tau_{\mathbb{S} \mathbb{C}}^{\tilde{\rho}}\left(\left[\alpha_{n}\right]_{1}\right) \cap \tau_{\mathbb{S} \mathbb{C}}^{\tilde{\rho}}\left(\left[\alpha_{n}\right]_{2}\right), \quad \tau_{\mathbb{S C}}^{\tilde{\sigma}}\left(\left[\alpha_{n}\right]_{1} \cap\left[\alpha_{n}\right]_{2}\right)>\tau_{\mathbb{S} \mathbb{C}}^{\tilde{\sigma}}\left(\left[\alpha_{n}\right]_{1}\right) \cup \tau_{\mathbb{S} \mathbb{C}}^{\tilde{\sigma}}\left(\left[\alpha_{n}\right]_{2}\right), \\
\tau_{\mathbb{S C}}^{\tilde{\varsigma}}\left(\left[\alpha_{n}\right]_{1} \cap\left[\alpha_{n}\right]_{2}\right)>\tau_{\mathbb{S} \mathbb{C}}^{\tilde{S}}\left(\left[\alpha_{n}\right]_{1}\right) \cup \tau_{\mathbb{S} \mathbb{C}}^{\tilde{\varsigma}}\left(\left[\alpha_{n}\right]_{2}\right)
\end{array}
$$

There exists $r \in \zeta_{0}$ such that

$$
\begin{array}{r}
\tau_{\mathbb{S} \mathbb{C}}^{\tilde{\rho}}\left(\left[\alpha_{n}\right]_{1} \cap\left[\alpha_{n}\right]_{2}\right)<r<\tau_{\mathbb{S C}}^{\tilde{\rho}}\left(\left[\alpha_{n}\right]_{1}\right) \cap \tau_{\mathbb{S C}}^{\tilde{\rho}}\left(\left[\alpha_{n}\right]_{2}\right), \quad \tau_{\mathbb{S C}}^{\tilde{\sigma}}\left(\left[\alpha_{n}\right]_{1} \cap\left[\alpha_{n}\right]_{2}\right)>1-r>\tau_{\mathbb{S C}}^{\tilde{\sigma}}\left(\left[\alpha_{n}\right]_{1}\right) \cup \tau_{\mathbb{S} \mathbb{C}}^{\tilde{\sigma}}\left(\left[\alpha_{n}\right]_{2}\right), \\
\tau_{\mathbb{S} \mathbb{C}}^{\tilde{S}}\left(\left[\alpha_{n}\right]_{1} \cap\left[\alpha_{n}\right]_{2}\right)>1-r>\tau_{\mathbb{S} \mathbb{C}}^{\tilde{S}}\left(\left[\alpha_{n}\right]_{1}\right) \cup \tau_{\mathbb{S C}}^{\tilde{S}}\left(\left[\alpha_{n}\right]_{2}\right)
\end{array}
$$

For each $i \in\{1,2\}$, there exists $r \in \zeta_{0}$ with $\mathbb{S C}\left(\left[\alpha_{n}\right]_{i}, r_{i}\right)=\left[\alpha_{n}\right]_{i}$ such that

$$
r<r_{i} \leq \tau_{\mathbb{S} \mathbb{C}}^{\tilde{\rho}}\left(\left[\alpha_{n}\right]_{i}\right), \quad \tau_{\mathbb{S} \mathbb{C}}^{\tilde{\sigma}}\left(\left[\alpha_{n}\right]_{i}\right) \leq 1-r_{i}<1-r, \quad \tau_{\mathbb{S C}}^{\tilde{s}}\left(\left[\alpha_{n}\right]_{i}\right) \leq 1-r_{i}<1-r .
$$

In addition, since $\mathbb{S C}_{\tau \tilde{\sigma} \tilde{\sigma} \tilde{\xi}}\left(\left[\alpha_{n}\right]_{i}, r\right)=\left[\alpha_{n}\right]_{i}$ by $\mathbb{S C}_{2}$ and $\mathbb{S C}_{4}$ of Definition 13 , for any $i \in\{1,2\}$,

$$
\mathbb{S C}_{\tau} \tilde{\tilde{\sigma} \tilde{\sigma} \tilde{\xi}}\left(\left[\alpha_{n}\right]_{1} \cup\left[\alpha_{n}\right]_{2}, r\right)=\left[\alpha_{n}\right]_{1} \cup\left[\alpha_{n}\right]_{2}
$$

It follows that $\tau_{\mathbb{S C}}^{\tilde{Q}}\left(\left[\alpha_{n}\right]_{1} \cap\left[\alpha_{n}\right]_{2}\right) \geq r, \tau_{\mathbb{S} \mathbb{C}}^{\tilde{\sigma}}\left(\left[\alpha_{n}\right]_{1} \cap\left[\alpha_{n}\right]_{2}\right) \leq 1-r$ and $\tau_{\mathbb{S} \mathbb{C}}^{\tilde{S}}\left(\left[\alpha_{n}\right]_{1} \cap\right.$ $\left.\left[\alpha_{n}\right]_{2}\right) \leq 1-r$. It is a contradiction. Thus, for every $\alpha_{n}, \varepsilon_{n} \in \zeta^{\mathcal{F}}, \tau_{\mathbb{S} \mathbb{C}}^{\tilde{Q}}\left(\alpha_{n} \cap \varepsilon_{n}\right) \geq \tau_{\mathbb{S} \mathbb{C}}^{\tilde{\rho}}\left(\alpha_{n}\right) \cap$ $\tau_{\mathbb{S} \mathbb{C}}^{\tilde{\rho}}\left(\varepsilon_{n}\right), \tau_{\mathbb{S} \mathbb{C}}^{\tilde{\sigma}}\left(\alpha_{n} \cap \varepsilon_{n}\right) \leq \tau_{\mathbb{S C}}^{\tilde{\sigma}}\left(\alpha_{n}\right) \cup \tau_{\mathbb{S} \mathbb{C}}^{\tilde{\sigma}}\left(\varepsilon_{n}\right)$ and $\tau_{\mathbb{S} \mathbb{C}}^{\tilde{S}}\left(\alpha_{n} \cap \varepsilon_{n}\right) \leq \tau_{\mathbb{S} \mathbb{C}}^{\tilde{S}}\left(\alpha_{n}\right) \cup \tau_{\mathbb{S} \mathbb{C}}^{\tilde{S}}\left(\varepsilon_{n}\right)$.

(SVNT3) Suppose that there exists $\alpha_{n}=\bigcup_{i \in \zeta},\left[\alpha_{n}\right]_{i} \in \zeta^{\mathcal{F}}$ such that

$$
\tau_{\mathbb{S} \mathbb{C}}^{\tilde{\rho}}\left(\alpha_{n}\right)<\bigcup_{i \in I} \tau_{\mathbb{S} \mathbb{C}}^{\tilde{\rho}}\left(\left[\alpha_{n}\right]_{i}\right), \quad \tau_{\mathbb{S} \mathbb{C}}^{\tilde{\sigma}}\left(\alpha_{n}\right)>\bigcup_{i \in I} \tau_{\mathbb{S} \mathbb{C}}^{\tilde{\tilde{C}}}\left(\left[\alpha_{n}\right]_{i}\right), \quad \tau_{\mathbb{S} \mathbb{C}}^{\tilde{\tau}}\left(\alpha_{n}\right)>\bigcup_{i \in I} \tau_{\mathbb{S} \mathbb{C}}^{\tilde{\tau}}\left(\left[\alpha_{n}\right]_{i}\right) .
$$

There exists $r_{0} \in \zeta_{0}$ such that

$$
\left.\tau_{\mathbb{S} \mathbb{C}}^{\tilde{\rho}}\left(\alpha_{n}\right)<r_{0}<\bigcup_{i \in I} \tau_{\mathbb{S} \mathbb{C}}^{\tilde{\rho}}\left(\left[\alpha_{n}\right]_{i}\right), \quad \tau_{\mathbb{S} \mathbb{C}}^{\tilde{\sigma}}\left(\alpha_{n}\right)>1-r_{0}>\bigcup_{i \in I} \tau_{\mathbb{S} \mathbb{C}}^{\tilde{\tau}}\left(\left[\alpha_{n}\right]_{i}\right), \quad \tau_{\mathbb{S} \mathbb{C}}^{\tilde{\tau}}\left(\alpha_{n}\right)\right)>1-r_{0}>\bigcup_{i \in I} \tau_{\mathbb{S} \mathbb{C}}^{\tilde{\tau}}\left(\left[\alpha_{n}\right]_{i}\right) .
$$

For every $i \in I$, there exists $\mathbb{S C}\left(\left[\alpha_{n}\right]_{i}^{c}, r_{i}\right)=\left[\alpha_{n}\right]_{i}^{c}$ and $r_{i} \in \zeta_{0}$ such that

$$
r_{0}<r_{i} \leq \tau_{\mathbb{S} \mathbb{C}}^{\tilde{\rho}}\left(\left[\alpha_{n}\right]_{i}\right), \quad 1-r_{0}>1-r_{i} \geq \tau_{\mathbb{S} \mathbb{C}}^{\tilde{\sigma}}\left(\left[\alpha_{n}\right]_{i}\right), \quad 1-r_{i}>1-r_{0} \geq \tau_{\mathbb{S} \mathbb{C}}^{\tilde{s}}\left(\left[\alpha_{n}\right]_{i}\right) .
$$

In addition, since $\mathbb{S C}\left(\left[\alpha_{n}\right]^{c}, r_{0}\right) \leq \mathbb{S C}\left(\left[\alpha_{n}\right]_{i}^{c}, r_{i}\right)=\left[\alpha_{n}\right]_{i}^{c}$, by $\mathbb{S C}_{2}$ of Definition 13 ,

$$
\mathbb{S C}\left(\left[\alpha_{n}\right]_{i}^{c}, r_{0}\right)=\left[\alpha_{n}\right]_{i}^{c}
$$

It implies, for all $i \in I$,

$$
\left.\mathbb{S C}\left(\alpha_{n}\right]^{c}, s_{0}\right) \leq \mathbb{S C}\left(\left[\alpha_{n}\right]_{i}^{c}, s_{0}\right)=\left[\alpha_{n}\right]_{i}^{c}
$$

It follows that

$$
\mathbb{S C}\left(\left[\alpha_{n}\right]^{c}, r_{0}\right) \leq \bigcap_{i \in J}\left(\left[\alpha_{n}\right]_{i}^{c}\right)=\left[\alpha_{n}\right]^{c} .
$$

Thus, $\mathbb{S C}\left(\left[\alpha_{n}\right]^{c}, r_{0}\right)=\left[\alpha_{n}\right]^{c}$, that is, $\tau_{\mathbb{S C}}^{\tilde{\rho}}\left(\alpha_{n}\right) \geq r_{0}, \tau_{\mathbb{S C}}^{\tilde{\sigma}}\left(\alpha_{n}\right) \leq 1-r_{0}$ and $\tau_{\mathbb{S} \mathbb{C}}^{\tilde{s}}\left(\alpha_{n}\right) \leq$ $1-r_{0}$. It is a contradiction. Hence, $\tau_{\mathbb{S} \mathbb{C}}^{\tau \tilde{C} \tilde{G}}$ is an $S V N T S$ on $\mathcal{F}$. 
Since $\alpha_{n} \leq \mathbb{S} \mathbb{C}\left(\alpha_{n}, r\right)$,

$\left.\tau_{\mathbb{S} \mathbb{C}}^{\tilde{\rho}}\left(\left[\mathbb{S C}\left(\alpha_{n}, r\right)\right]^{c}\right) \geq r, \tau_{\mathbb{S} \mathbb{C}}^{\tilde{S}}\left(\left[\mathbb{S C}\left(\alpha_{n}, r\right)\right]^{c}\right)\right) \leq 1-r, \tau_{\mathbb{S} \mathbb{C}}^{F}\left(\left[\mathbb{S C}\left(\alpha_{n}, r\right)\right]^{c}\right) \leq 1-r$.

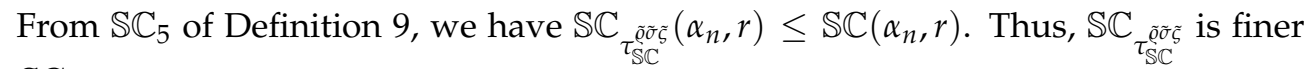
than $\mathbb{S C}$.

Example 1. Let $\mathcal{F}=\{a, b\}$. Define $\varepsilon_{n}, \pi_{n} \in \zeta^{\mathcal{F}}$ as follows:

$$
\varepsilon_{n}=\langle(0.1,0.1),(0.3,0.3),(0.3,0.3)\rangle ; \pi_{n}=\langle(0.4,0.4),(0.1,0.1),(0.1,0.1)\rangle .
$$

We define the mapping $\mathbb{S C}: \zeta^{\mathcal{F}} \times \zeta_{0} \rightarrow \zeta^{\mathcal{F}}$ as follows:

$$
\mathbb{S C}\left(\alpha_{n}, s\right)= \begin{cases}\tilde{0}, & \text { if } \alpha_{n}=\tilde{0}, \quad s \in \zeta_{0}, \\ \varepsilon_{n} \cap \pi_{n}, & \text { if } 0 \neq \alpha_{n} \leq \varepsilon_{n} \cap \pi_{n}, 0<r<\frac{1}{2}, \\ \varepsilon_{n}, & \text { if } \alpha_{n} \leq \varepsilon_{n}, \alpha_{n} \leq \pi_{n}, 0<r<\frac{1}{2}, \\ & \quad \text { or } 0 \neq \alpha_{n} \leq \varepsilon_{n} \quad \frac{1}{2}<r<\frac{2}{3}, \\ \pi_{n}, & \text { if } \alpha_{n} \leq \pi_{n}, \alpha_{n} \leq \varepsilon_{n}, 0<r<\frac{1}{2}, \\ \varepsilon_{n} \cup \pi_{n}, & \text { if } 0 \neq \alpha_{n} \leq \varepsilon_{n} \cup \pi_{n}, 0<r<\frac{1}{2}, \\ \tilde{1}, & \text { otherwise. }\end{cases}
$$

Then, $\mathbb{S C}$ is a single-valued neutrosophic closure operator.

From Theorem 2, we have a single-valued neutrosophic topology $\left(\tau_{\mathbb{S} \mathbb{C}}^{\tilde{\rho}}, \tau_{\mathbb{S} \mathbb{C}}^{\tilde{\sigma}}, \tau_{\mathbb{S} \mathbb{C}}^{\tilde{\varphi}}\right)$ on $\mathcal{F}$ as follows:

$$
\begin{aligned}
& \tau_{\mathbb{S} \mathbb{C}}^{\tilde{\rho}}\left(\alpha_{n}\right)= \begin{cases}1, & \text { if } \alpha_{n}=\tilde{1} \text { or } \tilde{0}, \\
\frac{2}{3}, & \text { if } \alpha_{n}=\left[\varepsilon_{n}\right]^{c}, \\
\frac{1}{2}, & \text { if } \alpha_{n}=\left[\pi_{n}\right]^{c}, \\
\frac{1}{2}, & \text { if } \alpha_{n}=\left[\varepsilon_{n}\right]^{c} \cup\left[\pi_{n}\right]^{c}, \\
\frac{1}{2}, & \text { if } \alpha_{n}=\left[\varepsilon_{n}\right]^{c} \cap\left[\pi_{n}\right]^{c}, \\
0, & \text { otherwise. }\end{cases} \\
& \tau_{\mathbb{S} \mathbb{C}}^{\tilde{\sigma}}\left(\alpha_{n}\right)= \begin{cases}0, & \text { if } \alpha_{n}=\tilde{1} \text { or } \tilde{0}, \\
\frac{1}{3}, & \text { if } \alpha_{n}=\left[\varepsilon_{n}\right]^{c}, \\
\frac{1}{2}, & \text { if } \alpha_{n}=\left[\pi_{n}\right]^{c}, \\
\frac{1}{2}, & \text { if } \alpha_{n}=\left[\varepsilon_{n}\right]^{c} \cup\left[\pi_{n}\right]^{c}, \\
\frac{1}{2}, & \text { if } \alpha_{n}=\left[\varepsilon_{n}\right]^{c} \cap\left[\pi_{n}\right]^{c}, \\
1, & \text { otherwise. }\end{cases} \\
& \tau_{\mathbb{S} \mathbb{C}}^{\tilde{\tau}}\left(\alpha_{n}\right)= \begin{cases}0, & \text { if } \alpha_{n}=\tilde{1} \text { or } \tilde{0}, \\
\frac{1}{3}, & \text { if } \alpha_{n}=\left[\varepsilon_{n}\right]^{c}, \\
\frac{1}{2}, & \text { if } \alpha_{n}=\left[\pi_{n}\right]^{c}, \\
\frac{1}{2}, & \text { if } \alpha_{n}=\left[\varepsilon_{n}\right]^{c} \cup\left[\pi_{n}\right]^{c}, \\
\frac{1}{2}, & \text { if } \alpha_{n}=\left[\varepsilon_{n}\right]^{c} \cap\left[\pi_{n}\right]^{c}, \\
1, & \text { otherwise. }\end{cases}
\end{aligned}
$$

Thus, the $\tau_{\mathbb{S} \mathbb{C}}^{\tilde{\rho} \tilde{\xi}}$ is a single-valued neutrosophic topology on $\mathcal{F}$.

Theorem 3. Let $\left(\tilde{\mathcal{F}}, \tau^{\tilde{\varrho} \tilde{\sigma} \tilde{S}}\right)$ be an SVNTS. Then, for any $\alpha_{n} \in \zeta^{\tilde{\mathcal{F}}}$ and $r \in \zeta_{0}$, we define an operator $\operatorname{SINT}_{\tau} \tau_{\tilde{\rho} \tilde{\sigma} \tilde{s}}: \zeta^{\tilde{\mathcal{F}}} \times \zeta_{0} \rightarrow \zeta^{\tilde{\mathcal{F}}}$ as follows:

$$
\operatorname{SINT}_{\tau \tilde{\varrho} \tilde{\sigma} \tilde{\zeta}}\left(\alpha_{n}, s\right)=\bigwedge\left\{\varepsilon_{n} \in \zeta^{\tilde{\mathcal{F}}}: \alpha_{n} \geq \varepsilon_{n}, \quad \varepsilon_{n} \text { is r-SVNSO }\right\} .
$$


For each $\alpha_{n}, \varepsilon_{n} \in \zeta^{\tilde{\mathcal{F}}}$ and $r, s \in \zeta_{0}$ the operator $\mathbb{S I N T}_{\tau} \tilde{\tilde{O} \tilde{\sigma} \tilde{\zeta}}$ satisfies the following conditions:

(1) $\operatorname{SINT}_{\tau} \tilde{\tau} \tilde{\sigma} \tilde{\tilde{\sigma}}(\tilde{1}, r)=\tilde{1}$,

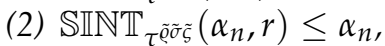

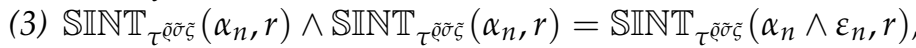

(4) $\mathbb{S I N T}_{\tau^{\tilde{\rho} \tilde{\sigma} \tilde{\zeta}}}\left(\alpha_{n}, r\right) \geq \mathbb{S I N T}_{\tau} \tilde{\tau} \tilde{\sigma} \tilde{\sigma}\left(\alpha_{n}, s\right)$ if $r \leq s$,

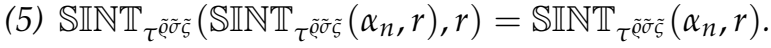

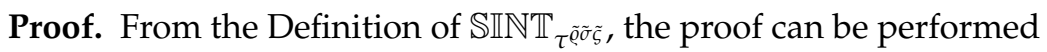

Definition 15. Let $\left(\tilde{\mathcal{F}}, \tau^{\tilde{\widetilde{\sigma}} \tilde{\mathcal{S}}}\right)$ be an SVNTS, $\alpha_{n} \in \zeta^{\tilde{\mathcal{F}}}, x_{s, t, k} \in \mathrm{P}_{s, t, k}(\tilde{\mathcal{F}})$ and $r \in \zeta_{0}$. Then,

(1) $\alpha_{n}$ is called $r$-single-valued neutrosophic $Q_{\tau}^{\tilde{Q} \tilde{\sigma} \tilde{s}}$-neighborhood of $x_{s, t, k}$ if $x_{s, t, k} q \alpha_{n}$ with

$$
\tau^{\tilde{\rho}}\left(\alpha_{n}\right) \geq r, \tau^{\tilde{\sigma}}\left(\alpha_{n}\right) \leq 1-r, \tau^{\tilde{S}}\left(\alpha_{n}\right) \leq 1-r,
$$

(2) $x_{s, t, k} \alpha_{n}$ is called $r$-single-valued neutrosophic $\theta$-cluster point (r- $\theta$-cluster point) of $\alpha_{n}$ if for any $\varepsilon_{n} \in Q_{\tilde{\tau} \tilde{\tau} \tilde{\sigma} \tilde{\zeta}}\left(x_{s, t, k}, r\right)$, we have $\alpha_{n} q \mathrm{C}_{\tilde{\tau} \tilde{\sigma} \tilde{\sigma} \tilde{\zeta}}\left(\varepsilon_{n}, r\right)$,

(3) $r$ - $\theta$-closure operator is a mapping $\mathrm{CI}_{\tilde{\tau} \tilde{\tau} \tilde{\sigma} \tilde{\zeta}}^{\theta}: \zeta^{\tilde{\mathcal{F}}} \times \zeta_{0} \rightarrow \zeta^{\tilde{\mathcal{F}}}$ defined as:

$$
\mathrm{C}_{\tilde{\tau} \tilde{\tilde{\sigma} \tilde{\sigma}} \tilde{\zeta}}^{\theta}\left(\alpha_{n}, r\right)=\bigvee\left\{x_{s, t, k} \in P_{s, t, k}(\tilde{\mathcal{F}}): x_{s, t, k} \text { is an } r-\theta \text {-cluster point of } \alpha_{n}\right\},
$$

(4) $\alpha_{n}$ is said to be $r$ - $\theta$-closed iff $\mathrm{CI}_{\tilde{\tau} \tilde{e} \tilde{\sigma} \tilde{\zeta}}^{\theta \in}\left(\alpha_{n}, r\right)=\alpha_{n}$. We define

$$
\Theta_{\tilde{\tau} \tilde{\sigma} \tilde{\sigma} \tilde{\zeta}}^{\theta}\left(\alpha_{n}, r\right)=\bigwedge\left\{\varepsilon_{n} \mid \alpha_{n} \leq \varepsilon_{n}, \varepsilon_{n}=\mathrm{CI}_{\tilde{\tau} \tilde{\rho} \tilde{\tilde{\sigma}} \tilde{c}}^{\theta}\left(\varepsilon_{n}, r\right)\right\} .
$$

Theorem 4. Let $\left(\tilde{\mathcal{F}}, \tau^{\tilde{\widetilde{\sigma}} \tilde{S}}\right)$ be an SVNTS. For $r \in \zeta_{0}$ and $\alpha_{n} \in \zeta^{\tilde{\mathcal{F}}}$. The following properties hold: (1) If $\alpha_{n} \leq \mathrm{C}_{\tilde{\tau} \tilde{\widetilde{\sigma} \tilde{S}}}^{\theta}\left(\alpha_{n}, r\right)$,

(2) If $\alpha_{n} \leq \varepsilon_{n}$, then $\mathrm{C}_{\tilde{\tau} \tilde{\tilde{\rho} \tilde{\sigma} \tilde{\sigma}}}^{\theta}\left(\alpha_{n}, r\right) \leq \mathrm{C}_{\tilde{\tau} \tilde{\tau} \tilde{\sigma} \tilde{\tilde{\sigma}}}^{\theta}\left(\varepsilon_{n}, r\right)$

(3) $\mathrm{C}_{\tilde{\tau} \tilde{\tilde{\sigma} \tilde{\sigma} \tilde{\zeta}}}^{\theta}\left(\alpha_{n}, r\right)=\bigwedge\left\{\varepsilon_{n} \mid \alpha_{n} \leq \operatorname{int}_{\tilde{\tau}}^{\theta} \tilde{\tilde{\sigma} \tilde{\sigma} \tilde{\zeta}}\left(\varepsilon_{n}, r\right), \tau^{\tilde{\varrho}}\left(\left[\varepsilon_{n}\right]^{c}\right) \geq r, \tau^{\tilde{\sigma}}\left(\left[\varepsilon_{n}\right]^{c}\right) \leq 1-r, \tau^{\tilde{S}}\left(\left[\varepsilon_{n}\right]^{c}\right) \leq\right.$ $1-r\}$.

(4) $\Theta_{\tilde{\tau} \tilde{\rho} \tilde{\sigma} \tilde{\sigma}}^{\theta}\left(\alpha_{n}, r\right)=\mathrm{CI}_{\tilde{\tau} \tilde{\rho} \tilde{\sigma} \tilde{\sigma}}^{\theta}\left(\Theta_{\tilde{\tau} \tilde{\sigma} \tilde{\sigma} \tilde{\bar{s}}}^{\delta}\left(\alpha_{n}, r\right), r\right)$,

(5) $\Theta_{\tilde{\tau} \tilde{\varphi} \tilde{\sigma} \tilde{\xi}}^{\theta}\left(\alpha_{n}, r\right)$ is $r-\theta$-closed,

(6) $\mathrm{CI}_{\tilde{\tau} \tilde{\varphi} \tilde{\sigma} \tilde{\zeta}}^{\theta}\left(\alpha_{n}, r\right) \leq \Theta_{\tilde{\tau} \tilde{\tilde{\sigma} \tilde{\zeta}} \tilde{\tilde{s}}}^{\theta}\left(\alpha_{n}, r\right)$.

Proof. (1) and (2) are easily proved from Definition 14

(3) $\gamma=\bigwedge\left\{\varepsilon_{n} \mid \alpha_{n} \leq \operatorname{int}_{\tilde{\tau} \tilde{\sigma} \tilde{\sigma}}^{\theta}\left(\varepsilon_{n}, r\right), \tau^{\tilde{\rho}}\left(\left[\varepsilon_{n}\right]^{c}\right) \geq r, \tau^{\tilde{\sigma}}\left(\left[\varepsilon_{n}\right]^{c}\right) \leq 1-r, \tau^{\tilde{S}}\left(\left[\varepsilon_{n}\right]^{c}\right) \leq 1-r\right\}$. Suppose that $C_{\tilde{\tau} \tilde{\rho} \tilde{\tilde{\sigma}} \tilde{\xi}}^{\theta}\left(\alpha_{n}, r\right) \geq \gamma$, then there exists $v \in \mathcal{F}$ and $s, t, k \in \zeta_{0}$ such that

$$
\begin{aligned}
& \tau_{\mathrm{C}_{\tilde{\tau} \tilde{\rho}}^{\theta}\left(\alpha_{n}, r\right)}^{\tilde{\rho}}(v)<s \leq \tau_{\gamma}^{\tilde{\rho}}(v) . \\
& \tau_{\mathrm{C}_{\tilde{\tau} \tilde{\sigma}}^{\theta}\left(\alpha_{n}, r\right)}^{\tilde{\sigma}}(v)>t \geq \tau_{\gamma}^{\tilde{\sigma}}(v) \\
& \tau_{\mathrm{C}_{\tilde{\tau} \tilde{\zeta}}^{\theta}\left(\alpha_{n}, r\right)}^{\tilde{\rho}}(v)>k \geq \tau_{\gamma}^{\tilde{\zeta}}(v) .
\end{aligned}
$$

Then $x_{S, t, K}$ is not $r$ - $\theta$-cluster point of $\alpha_{n}$. So, there exists $\varepsilon_{n} \in Q_{\tau} \tilde{e} \tilde{\sigma} \tilde{\sigma}\left(x_{s, t, k}, r\right)$, and $\alpha_{n} \leq$ $\left[\mathrm{C}_{\tilde{\tau} \tilde{\rho} \tilde{\sigma} \tilde{\sigma}}\left(\left[\varepsilon_{n}, r\right)\right]^{c}\right.$. Thus, $\alpha_{n} \leq\left[\mathrm{C}_{\tilde{\tau} \tilde{\sigma} \tilde{\sigma} \tilde{\zeta}}\left(\varepsilon_{n}, r\right)\right]^{c}=\operatorname{int}_{\tilde{\tau} \tilde{\sigma} \tilde{\sigma} \tilde{\zeta}}\left(\left[\varepsilon_{n}\right]^{c}, r\right)$ and $\tau^{\tilde{\rho}}\left(\varepsilon_{n}\right) \geq r, \tau^{\tilde{\sigma}}\left(\varepsilon_{n}\right) \leq 1-r$, $\tau \tilde{\zeta}\left(\varepsilon_{n}\right) \leq 1-r$. Hence,

$$
\tau_{\gamma}^{\tilde{\rho}}(v) \leq \tau_{\left.\left[\varepsilon_{n}\right)\right]}^{\tilde{\rho}}(v)<s, \quad \tau_{\gamma}^{\tilde{\sigma}}(v) \geq \tau_{\left.\left[\varepsilon_{n}\right)\right]^{c}}^{\tilde{\rho}}(v)>t, \quad \tau_{\gamma}^{\tilde{\zeta}}(v) \geq \tau_{\left.\left[\varepsilon_{n}\right)\right]^{c}}^{\tilde{\varphi}}(v)>k .
$$

It is a contradiction for Equation (1). Thus $C_{\tilde{\tau} \tilde{\rho} \tilde{\tilde{\sigma}} \tilde{\sigma}}^{\theta}\left(\alpha_{n}, r\right) \geq \gamma$. 
Suppose that $C_{\tilde{\tau} \tilde{\rho} \tilde{\sigma} \tilde{G}}^{\theta}\left(\alpha_{n}, r\right) \not z$, then there exists $r$ - $\theta$-cluster point of $y_{s, t, k} \in P_{x_{s, t, k}}(\tilde{\mathcal{F}})$ of $\alpha_{n}$ such that

$$
\begin{aligned}
& \tau_{\mathrm{C}_{\tilde{\tau} \tilde{\rho}}^{\theta}\left(\alpha_{n}, r\right)}^{\tilde{\rho}}(y)>s \geq \tau_{\gamma}^{\tilde{\rho}}(y) . \\
& \tau_{\mathrm{C}_{\tilde{\tau} \tilde{\sigma}}^{\tilde{\theta}}\left(\alpha_{n}, r\right)}^{\tilde{\sigma}}(y)<t \leq \tau_{\gamma}^{\tilde{\sigma}}(y) \\
& \tau_{\mathrm{C}_{\tilde{\tau} \tilde{\tilde{s}}}^{\theta}\left(\alpha_{n}, r\right)}^{\tilde{\sigma}}(y)<k \leq \tau_{\gamma}^{\tilde{\zeta}}(v) .
\end{aligned}
$$

By definition of $\gamma$, there exists $\varepsilon_{n} \in \zeta^{\text {tilde } \mathcal{F}}$ with $\alpha_{n} \leq \operatorname{int}_{\tilde{\tau}} \tilde{\tilde{\sigma} \tilde{\sigma} \tilde{S}}\left(\varepsilon_{n}, r\right)$ and $\tau^{\tilde{\varrho}}\left(\left[\varepsilon_{n}\right]^{c}\right) \geq r$, $\tau^{\tilde{\sigma}}\left(\left[\varepsilon_{n}\right]^{c}\right) \leq 1-r, \tau^{\tilde{S}}\left(\left[\varepsilon_{n}\right]^{c}\right) \leq 1-r$ such that

$$
\begin{aligned}
& \tau_{\mathrm{C}_{\tilde{\tau}}^{\theta} \tilde{\rho}\left(\alpha_{n}, r\right)}^{\tilde{\rho}}(y)>s>\tau_{\varepsilon_{n}}^{\tilde{\rho}}(y) \geq \tau_{\gamma}^{\tilde{\rho}}(y), \\
& \tau_{\mathrm{C}_{\tilde{\tau} \tilde{\sigma}}^{\tilde{\sigma}}\left(\alpha_{n}, r\right)}^{\tilde{\theta}}(y)<t<\tau_{\varepsilon_{n}}^{\tilde{\sigma}}(y) \leq \tau_{\gamma}^{\tilde{\sigma}}(y), \\
& \tau_{\mathrm{C}_{\tilde{\tau} \tilde{\zeta}}^{\theta}\left(\alpha_{n}, r\right)}^{\tilde{\zeta}}(y)<k<\tau_{\varepsilon_{n}}^{\tilde{\zeta}}(y) \leq \tau_{\gamma}^{\tilde{\zeta}}(v) .
\end{aligned}
$$

Then $\left[\varepsilon_{n}\right]^{c} \in Q_{\tau} \tilde{\tilde{\rho} \tilde{\sigma} \tilde{s}}\left(y_{s, t, k}, r\right)$. Furthermore, $\alpha_{n} \leq \operatorname{int}_{\tilde{\tau} \tilde{\tilde{\rho}} \tilde{\tilde{\rho}} \tilde{\tilde{s}}}\left(\varepsilon_{n}, r\right)=\left[\mathrm{C}_{\tilde{\tau} \tilde{\rho} \tilde{\sigma} \tilde{\tilde{s}}}\left(\left[\varepsilon_{n}\right]^{c}, r\right)\right]^{c}$ which implies $\alpha_{n} \bar{q} C_{\tilde{\tau} \tilde{\rho} \tilde{\sigma} \tilde{\xi}}\left(\left[\varepsilon_{n}\right]^{c}, r\right)$. Hence $y_{s, t, t, k}$ is not an $r$ - $\theta$-cluster point of $\alpha_{n}$. It is a contradiction for Equation (2). Thus $\mathrm{C}_{\tilde{\tau} \tilde{\rho} \tilde{\sigma} \tilde{\xi}}^{\theta}\left(\alpha_{n}, r\right) \leq \gamma$.

(4) Let $\alpha_{n} \leq\left[\varepsilon_{n}\right]_{i}=\mathrm{CI}_{\tilde{\tau} \tilde{\tilde{\sigma} \tilde{\sigma} \tilde{\xi}}}^{\theta}\left(\left[\varepsilon_{n}\right]_{i}, r\right)$ for each $i \in \Gamma$. Then

$$
\bigwedge_{i \in \Gamma}\left[\varepsilon_{n}\right]_{i} \leq \mathrm{C}_{\tilde{\tau} \tilde{\tilde{\sigma} \tilde{\sigma} \tilde{\zeta}}}^{\theta}\left(\bigwedge_{i \in \Gamma}\left[\varepsilon_{n}\right]_{i}, r\right) \leq \mathrm{C}_{\tilde{\tau} \tilde{\tau} \tilde{\sigma} \tilde{\zeta}}^{\theta}\left(\left[\mathcal{E}_{n}\right]_{i}, r\right)=\left[\varepsilon_{n}\right]_{i} .
$$

So, $\bigwedge_{i \in \Gamma}\left[\varepsilon_{n}\right]_{i} \leq \mathrm{C}_{\tilde{\tau} \tilde{\rho} \tilde{\sigma} \tilde{G}}^{\theta}\left(\bigwedge_{i \in \Gamma}\left[\varepsilon_{n}\right]_{i}, r\right)$. Hence, $\Theta_{\tilde{\tau} \tilde{\sigma} \tilde{\sigma} \tilde{\zeta}}^{\theta}\left(\alpha_{n}, r\right)=\mathrm{CI}_{\tilde{\tau} \tilde{\widetilde{\sigma} \tilde{\zeta}}}^{\theta}\left(\Theta_{\tilde{\tau} \tilde{\sigma} \tilde{\sigma} \tilde{\zeta}}^{\theta \in}\left(\alpha_{n}, r\right), r\right)$.

(5) It is directly obtained from (4).

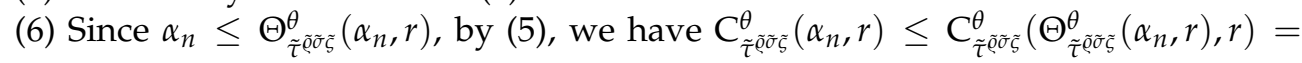
$\Theta_{\tilde{\tau} \tilde{\tilde{o} \tilde{\zeta}}}^{\theta}\left(\alpha_{n}, r\right)$.

Definition 16. Let $\left(\tilde{\mathcal{F}}, \tau^{\tilde{\tilde{\sigma}} \tilde{\zeta}}, £^{\tilde{\sigma} \tilde{\sigma} \tilde{S}}\right)$ be an SVNITS and $\alpha_{n} \in \zeta^{\tilde{\mathcal{F}}}, r \in \zeta_{0}$. Then, $\alpha_{n}$ is said to be $r$ -

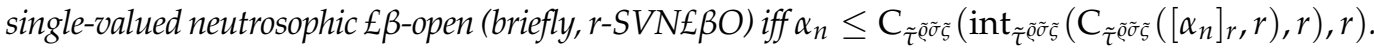
The complement of $r$-SVN $\beta O$ is said to be $r-S V N \beta C$.

Remark 2. Let $\left(\tilde{\mathcal{F}}, \tau^{\tilde{\rho} \tilde{\sigma} \tilde{S}}, £^{\tilde{\sigma} \tilde{\sigma} \tilde{S}}\right)$ be an SVNITS. For $\alpha_{n} \in \zeta^{\tilde{\mathcal{F}}}, x_{s, t, k} \in \mathrm{P}_{x_{s, t, k}}(\tilde{\mathcal{F}})$ and $r \in \zeta_{0}$. Then,

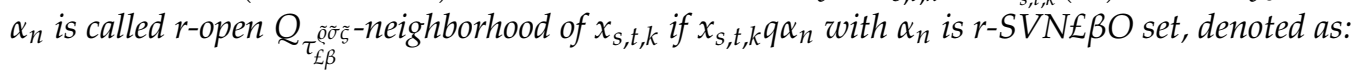

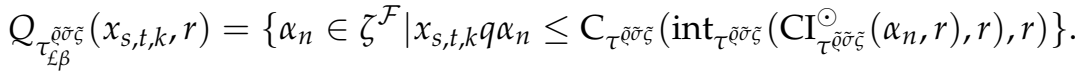

Definition 17. Let $\left(\tilde{\mathcal{F}}, \tau^{\tilde{\tilde{\sigma}} \tilde{S}}, £^{\tilde{\tilde{\sigma}} \tilde{\sigma} \tilde{S}}\right)$ be an SVNITS. For each $\alpha_{n}, \varepsilon_{n} \in \zeta^{\tilde{\mathcal{F}}}$ and $r \in \zeta_{0}$, we define the

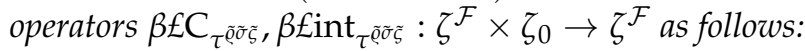

$$
\begin{aligned}
\beta £ \mathrm{C}_{\tau \tilde{\tau} \tilde{\sigma} \tilde{\zeta}}\left(\alpha_{n}, r\right) & =\bigwedge\left\{\varepsilon_{n} \in \zeta^{\mathcal{F}} \mid \alpha_{n} \leq \varepsilon_{n}, \varepsilon_{n} \text { is r-SVN } £ \beta C\right\}, \\
\beta \text { int }_{\tau} \tilde{\sigma} \tilde{\sigma} \tilde{\zeta} & \left(\alpha_{n}, r\right)=\bigvee\left\{\varepsilon_{n} \in \zeta^{\mathcal{F}} \mid \varepsilon_{n} \leq \alpha_{n}, \varepsilon_{n} \text { is r-SVN } £ \beta \mathrm{O}\right\} .
\end{aligned}
$$

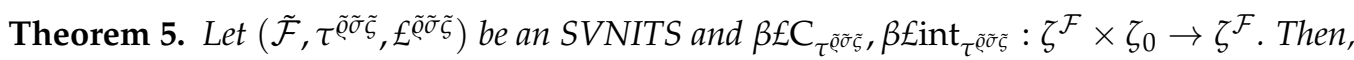


(1) $\beta \notin \mathrm{C}_{\tau} \tilde{\tilde{\rho} \tilde{\sigma} \tilde{\zeta}}(\tilde{0}, r)=\tilde{0}$,

(2) $\alpha_{n} \leq \beta \notin C_{\tau} \tilde{\sigma} \tilde{\sigma} \tilde{\xi}\left(\alpha_{n}, r\right)$,

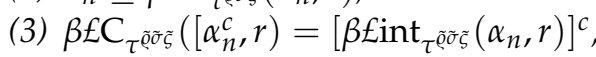

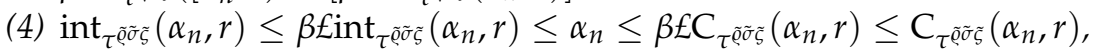

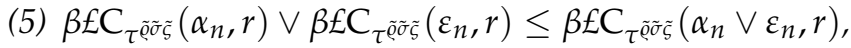

(6) $\alpha_{n}$ is r-SVN $£ \beta C$ iff $\alpha_{n}=\beta £ \mathrm{C}_{\tau} \tilde{\tilde{\rho} \tilde{\sigma} \tilde{\zeta}}\left(\alpha_{n}, r\right)$,

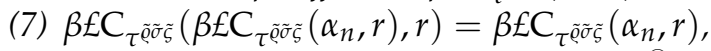

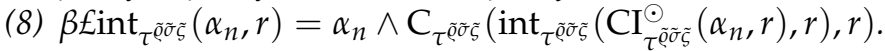

Proof. (1), (2), (3), (4), (5) and (6) are easily proved from the definitions of $\beta \notin C_{\tau} \tilde{\sigma \tilde{\sigma} \tilde{\sigma} \tilde{S}}$ and $\beta \notin$ int $_{\tau} \tilde{\tau} \tilde{\sigma} \tilde{\sigma}$.

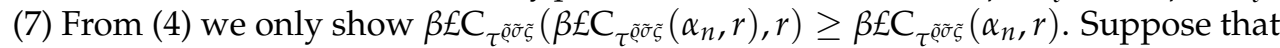

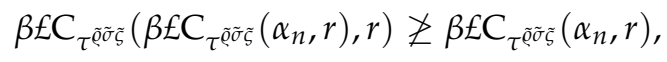

there exist $v \in \mathcal{F}$ and $s, t, k \in \zeta_{0}$ such that

$$
\begin{aligned}
& \tau_{\beta \notin \mathrm{C}_{\tau} \tilde{\rho}\left(\alpha_{n}, r\right)}^{\tilde{\rho}}(v)<s<\tau_{\beta \notin \mathrm{C}_{\tau \tilde{\rho}}^{\tilde{\rho}}\left(\beta \notin \mathrm{C}_{\tau \tilde{\rho}}^{\tilde{\rho}}\left(\alpha_{n}, r\right), r\right)}(v),
\end{aligned}
$$

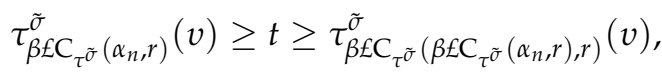

$$
\begin{aligned}
& \tau_{\beta \notin C_{\tau \tilde{\zeta}}\left(\alpha_{n}, r\right)}^{\tilde{\zeta}}(v) \geq k \geq \tau_{\beta \in C_{\tau \tilde{\zeta}}\left(\beta \notin C_{\tau \tilde{\zeta}}\left(\alpha_{n}, r\right), r\right)}(v),
\end{aligned}
$$

Since $\tau_{\beta \notin C_{\tau} \tilde{\rho}\left(\alpha_{n}, r\right)}^{\tilde{\rho}}(v)<s, \tau_{\beta \notin C_{\tau \tilde{\sigma}}\left(\alpha_{n}, r\right)}^{\tilde{\sigma}}(v) \geq t$ and $\tau_{\beta \notin C_{\tau} \tilde{\zeta}\left(\alpha_{n}, r\right)}^{\tilde{\zeta}}(v) \geq k$ by definition $\beta £ C_{\tau \tilde{\sigma} \tilde{\sigma} \tilde{\zeta}}$ there exists $r$-SVN£ßC set $\varepsilon_{n} \in \zeta^{\mathcal{F}}$ with $\alpha_{n} \leq \varepsilon_{n}$ such that

$$
\begin{aligned}
& \tau_{\beta \notin \mathrm{C}_{\tau \tilde{\rho}}\left(\alpha_{n}, r\right)}^{\tilde{\rho}}(v) \leq \tau_{\varepsilon_{n}}^{\tilde{\rho}}(v)<s, \\
& \tau_{\beta \notin \mathrm{C}_{\tau^{\tilde{\sigma}}}\left(\alpha_{n}, r\right)}^{\tilde{\sigma}}(v) \geq \tau_{\varepsilon_{n}}^{\tilde{\sigma}}(v) \geq t, \\
& \tau_{\beta \notin \mathrm{C}_{\tau \tilde{\zeta}}\left(\alpha_{n}, r\right)}^{\tilde{\zeta}}(v) \geq \tau_{\varepsilon_{n}}^{\tilde{\xi}}(v) \geq k .
\end{aligned}
$$

Since $\alpha_{n} \leq \varepsilon_{n}, \beta \notin C_{\tau \tilde{\rho} \tilde{\sigma} \tilde{\xi}}\left(\alpha_{n}, r\right) \leq \varepsilon_{n}$. Again, by the definition of $\beta \mathcal{I} C_{\tau}$, we have $\beta \notin \mathrm{C}_{\tau \tilde{\rho} \tilde{\rho} \tilde{s}}\left(\beta £ \mathrm{C}_{\tau \tilde{\rho} \tilde{\sigma} \tilde{\zeta}}\left(\alpha_{n}, r\right), r\right) \leq \varepsilon_{n}$. Hence

$$
\begin{aligned}
& \tau_{\beta \notin C_{\tau^{\tilde{\rho}}}^{\tilde{\rho}}\left(\beta \notin \mathrm{C}_{\tau \tilde{\rho}}\left(\alpha_{n}, r\right), r\right)}(v) \leq \tau_{\mathcal{E}_{n}}^{\tilde{\rho}}(v)<s, \\
& \tau_{\beta \notin C_{\tau^{\tilde{\sigma}}}^{\tilde{\sigma}}\left(\beta \notin \mathrm{C}_{\tau^{\tilde{\sigma}}}\left(\alpha_{n}, r\right), r\right)}(v) \geq \tau_{\varepsilon_{n}}^{\tilde{\sigma}}(v) \geq t, \\
& \tau_{\beta \notin C_{\tau \tilde{\zeta}}^{\tilde{\zeta}}\left(\beta \notin C_{\tau \tilde{\zeta}}\left(\alpha_{n}, r\right), r\right)}(v) \geq \tau_{\mathcal{E}_{n}}^{\tilde{\zeta}}(v) \geq k .
\end{aligned}
$$

It is a contradiction for Equation (1).

(8) Since

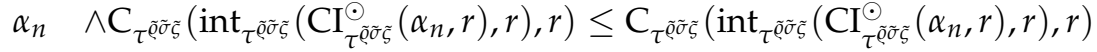

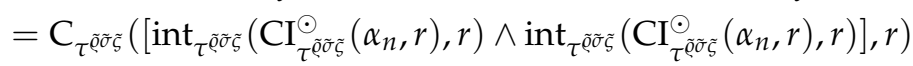

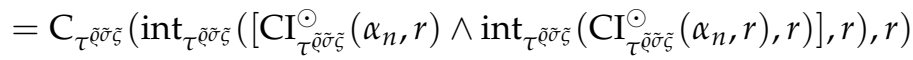

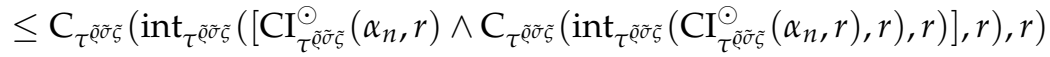

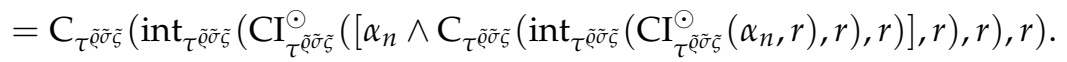




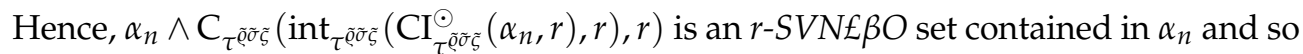

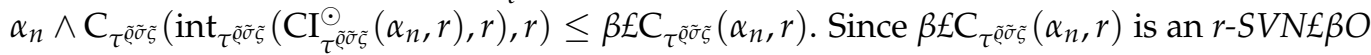
set, we have

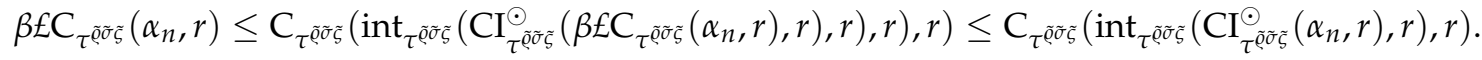

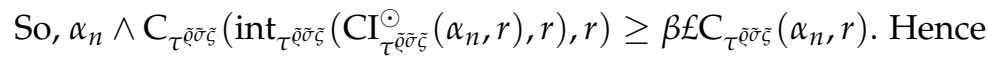

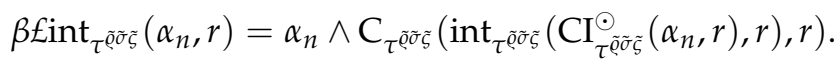

\section{New Terms of Single-Valued Neutrosophic Continuity}

In this section, we introduce and characterize new classes of mappings called singlevalued neutrosophic almost $\beta £$, faintly $\beta £$, weakly $\beta £$ and $\beta £$-continuous mappings. These findings lead to many theorems and consequences. Using these different attributes, we provide an example at the end of this section to show the difference between these kinds of mappings.

Definition 18. Let $f:\left(\tilde{\mathcal{F}}, \tau^{\tilde{\varphi} \widetilde{\sigma} \tilde{S}}, £^{\tilde{\sigma} \tilde{\sigma} \tilde{S}}\right) \rightarrow\left(\tilde{\mathcal{G}}, \varphi^{\tilde{\sigma} \tilde{\sigma} \tilde{S}}\right)$ be a mapping. Then, $f$ is called singlevalued neutrosophic almost $\beta €$-continuous mapping (SVNA $£ \subset$, for short) iff for each $\alpha_{n} \in$

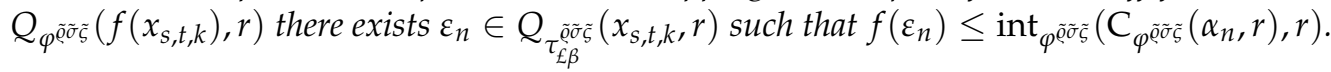

Lemma 1. For any single-valued neutrosophic set $\alpha_{n} \in \zeta^{\mathcal{F}}$ in an $\operatorname{SVNTS}\left(\tilde{\mathcal{F}}, \tau^{\tilde{\sigma} \tilde{\sigma} \tilde{S}}\right)$ and $r \in \zeta_{0}$,

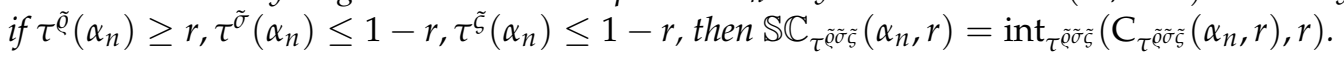

Lemma 2. For any single-valued neutrosophic set $\alpha_{n} \in \zeta^{\mathcal{F}}$ in an $\operatorname{SVNTS}\left(\tilde{\mathcal{F}}, \tau^{\tilde{Q} \tilde{\sigma} \tilde{S}}\right)$ and $r \in \zeta_{0}$,

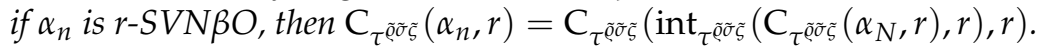

Theorem 6. Let $f:\left(\tilde{\mathcal{F}}, \tau^{\tilde{\tilde{\sigma}} \tilde{\zeta}}, £^{\tilde{\rho} \tilde{\sigma} \tilde{S}}\right) \rightarrow\left(\tilde{\mathcal{G}}, \varphi^{\tilde{\tilde{\sigma}} \tilde{\mathcal{S}}}\right)$ be a mapping for each $\alpha_{n} \in \zeta^{\tilde{\mathcal{G}}}$ and $r \in \zeta_{0}$. Then the following statements are equivalent:

(1) $f$ is SVNAB£C,

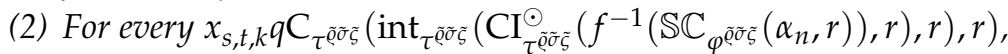

(3) $f^{-1}\left(\alpha_{n}\right) \leq \beta$ fint $_{\tau^{\tilde{\rho} \tilde{\sigma} \tilde{\zeta}}}\left(f^{-1}\left(\mathbb{S C}_{\varphi^{\tilde{\rho} \tilde{\sigma} \tilde{\zeta}}}\left(\alpha_{n}, r\right)\right), r\right)$ for every $\varphi^{\tilde{\rho}}\left(\alpha_{n}\right) \geq r, \varphi^{\tilde{\sigma}}\left(\alpha_{n}\right) \leq 1-r, \varphi^{\tilde{S}}\left(\alpha_{n}\right)$ $\leq 1-r$.

Proof. (1) $\Rightarrow(2)$ : Let $\alpha_{n} \in Q_{\varphi^{\tilde{Q} \tilde{\sigma} \tilde{G}}}\left(f\left(x_{s, t, k}\right), r\right)$. Then by (1), there exists $\varepsilon_{n} \in Q_{\tau_{\tilde{\epsilon}}^{\tilde{\tilde{\sigma}} \tilde{\xi}}}\left(x_{s, t, k}, r\right)$ such that $f\left(\varepsilon_{n}\right) \leq \operatorname{int}_{\phi \tilde{\sigma} \tilde{\sigma} \tilde{\zeta}}\left(\mathrm{C}_{\varphi \tilde{\sigma} \tilde{\sigma} \tilde{\zeta}}\left(\alpha_{n}, r\right), r\right)$. Since, $\varphi^{\tilde{\varrho}}\left(\alpha_{n}\right) \geq r, \varphi^{\tilde{\sigma}}\left(\alpha_{n}\right) \leq 1-r, \varphi^{\tilde{S}}\left(\alpha_{n}\right) \leq 1-r$. by Lemma 1, $\mathbb{S C}_{\varphi^{\tilde{\sigma} \tilde{\sigma} \tilde{\xi}}}\left(\alpha_{n}, r\right)=\operatorname{int}_{\varphi^{\tilde{\sigma} \tilde{\sigma} \tilde{\xi}}}\left(\mathrm{C}_{\varphi^{\tilde{\sigma}} \tilde{\sigma} \tilde{\bar{\zeta}}}\left(\alpha_{n}, r\right), r\right)$. Hence, $f\left(\varepsilon_{n}\right) \leq \operatorname{SSC}_{\varphi^{\tilde{\rho} \tilde{\sigma} \tilde{\xi}}}\left(\alpha_{n}, r\right)$. Since $\varepsilon_{n}$ is $r-S V N £ \beta O$ set,

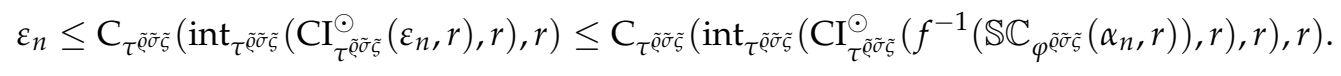

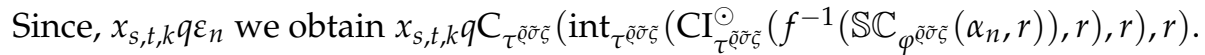

$(2) \Rightarrow(3)$ : Let $x_{s, t, k} q f^{-1}\left(\alpha_{n}\right)$ and $\varphi^{\tilde{\varrho}}\left(\alpha_{n}\right) \geq r, \varphi^{\tilde{\sigma}}\left(\alpha_{n}\right) \leq 1-r, \varphi^{\tilde{S}}\left(\alpha_{n}\right) \leq 1-r$, by (2), we have

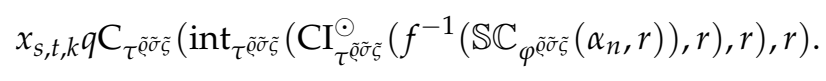

Since $x_{s, t, k} q f^{-1}\left(\alpha_{n}\right)$ we obtain $x_{s, t, k} q f^{-1}\left(\mathbb{S C}_{\varphi \tilde{o} \tilde{\sigma} \tilde{\xi}}\left(\alpha_{n}, r\right)\right)$. Hence, by Theorem 5(8),

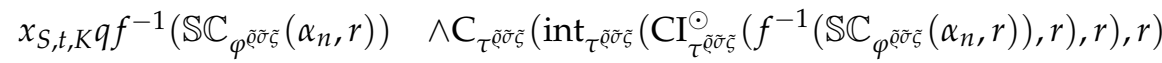

$$
\begin{aligned}
& =\beta \notin \operatorname{int}_{\tau} \tilde{\tilde{\sigma} \tilde{\sigma} \tilde{\zeta}}\left(f^{-1}\left(\mathbb{S C}_{\varphi^{\tilde{\rho} \tilde{\sigma} \tilde{\zeta}}}\left(\alpha_{n}, r\right)\right), r\right) \text {. }
\end{aligned}
$$


Thus, $x_{S, t, K} q f^{-1}\left(\alpha_{n}\right)$ implies $x_{S, t, K} q \beta \operatorname{int}_{\tau \tilde{\rho} \tilde{\sigma} \tilde{\sigma}}\left(f^{-1}\left(\mathbb{S C}_{\varphi_{\varphi} \tilde{\sigma} \tilde{\sigma} \tilde{S}}\left(\alpha_{n}, r\right)\right), r\right)$. Hence

$$
f^{-1}\left(\alpha_{n}\right) \leq \beta \notin \operatorname{int}_{\tau} \tilde{\tilde{\rho} \tilde{\sigma} \tilde{G}}\left(f^{-1}\left(\mathbb{S C}_{\varphi^{\tilde{Q} \tilde{\sigma} \tilde{G}}}\left(\alpha_{n}, r\right)\right), r\right) .
$$

$(3) \Rightarrow(1)$ : Let $\alpha_{n} \in Q_{\varphi^{\tilde{\sigma} \tilde{\sigma} \tilde{S}}}\left(f\left(x_{s, t, k}\right), r\right)$. and since $f^{-1}\left(\alpha_{n}\right) \leq \beta \notin \operatorname{int}_{\tau} \tau_{\tilde{\sigma} \tilde{\sigma} \tilde{S}}\left(f^{-1}\left(\mathbb{S C}_{\varphi^{\tilde{\sigma} \tilde{\sigma} \tilde{\zeta}}}\left(\alpha_{n}, r\right)\right)\right.$,

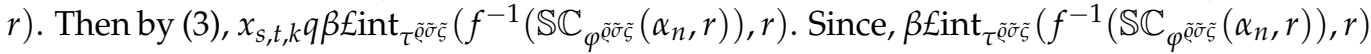

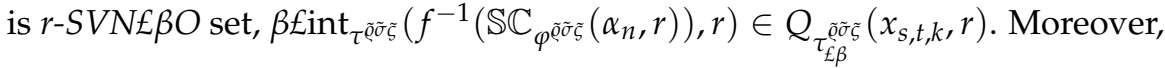

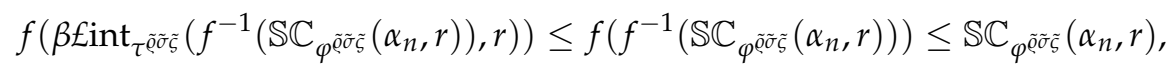

by Lemma 1, $f\left(\beta £ \operatorname{int}_{\tau^{\tilde{\sigma} \tilde{\sigma} \tilde{\zeta}}}\left(f^{-1}\left(\mathbb{S C}_{\varphi^{\tilde{\sigma} \tilde{\sigma} \tilde{\zeta}}}\left(\alpha_{n}, r\right)\right), r\right)\right) \leq \operatorname{int}_{\varphi^{\tilde{r} \tilde{\sigma} \tilde{\zeta}}}\left(\mathrm{C}_{\varphi^{\tilde{\sigma} \tilde{\sigma} \tilde{\zeta}}}\left(\alpha_{n}, r\right), r\right)$.

Theorem 7. Let $f:\left(\tilde{\mathcal{F}}, \tau^{\tilde{\varrho} \tilde{\sigma} \tilde{S}}, £^{\tilde{\sigma} \tilde{\mathcal{\sigma}} \tilde{S}}\right) \rightarrow\left(\tilde{\mathcal{G}}, \varphi^{\tilde{Q} \tilde{\sigma} \tilde{S}}\right)$ be a mapping for each $\alpha_{n} \in \zeta^{\tilde{\mathcal{G}}}, \varepsilon_{n} \in \zeta^{\tilde{\mathcal{F}}}$ and $r \in \zeta_{0}$. Then the following statements are equivalent:

(1) $f$ is $S V N A \beta E C$,

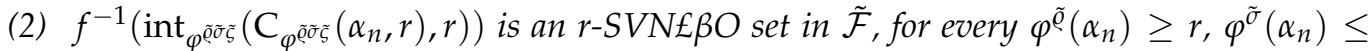
$1-r, \varphi \tilde{s}\left(\alpha_{n}\right) \leq 1-r$

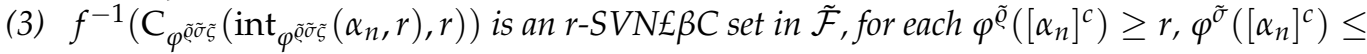
$1-r, \varphi \tilde{s}\left(\left[\alpha_{n}\right]^{c}\right) \leq 1-r$,

(4) $f^{-1}\left(\alpha_{n}\right)$ is an $r$-SVN£ßO set in $\tilde{\mathcal{F}}$, for each $r$-SVNRO set $\alpha_{n} \in \zeta^{\tilde{\mathcal{G}}}$,

(5) $f^{-1}\left(\alpha_{n}\right)$ is an $r-S V N £ \beta C$ set in $\tilde{\mathcal{F}}$, for each $r$-SVNRC set $\alpha_{n} \in \zeta^{\tilde{\mathcal{G}}}$,

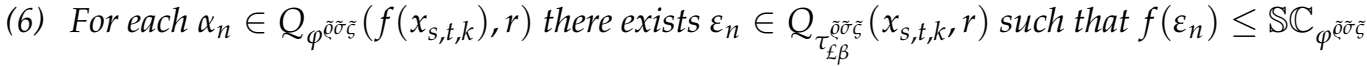
$\left(\alpha_{n}, r\right)$,

(7) $f^{-1}\left(\alpha_{n}\right) \leq \beta \operatorname{fint}_{\tau \tilde{\varrho} \tilde{\sigma} \tilde{\zeta}}\left(f^{-1}\left(\mathbb{S C}_{\varphi} \varphi_{\tilde{\sigma} \tilde{\sigma} \tilde{\zeta}}\left(\alpha_{n}, r\right)\right), r\right)$ for each $\varphi^{\tilde{\rho}}\left(\alpha_{n}\right) \geq r, \varphi^{\tilde{\sigma}}\left(\alpha_{n}\right) \leq 1-r, \varphi^{\tilde{S}}\left(\alpha_{n}\right)$ $\leq 1-r$,

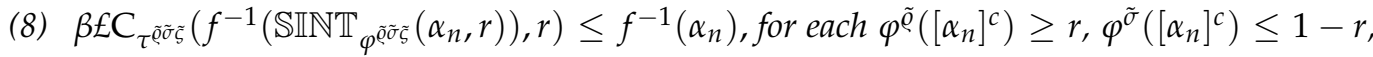
$\varphi^{\tilde{S}}\left(\left[\alpha_{n}\right]^{c}\right) \leq 1-r$,

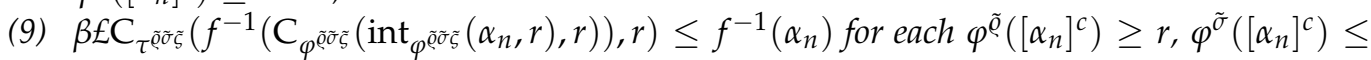
$1-r, \varphi^{\tilde{S}}\left(\left[\alpha_{n}\right]^{c}\right) \leq 1-r$,

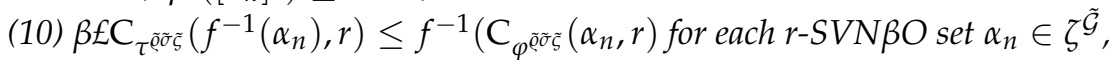

(11) $\beta \notin \mathrm{C}_{\tau} \tilde{\tilde{\sigma} \tilde{\sigma} \tilde{\zeta}}\left(f^{-1}\left(\alpha_{n}\right), r\right) \leq f^{-1}\left(\mathrm{C}_{\phi \tilde{\sigma} \tilde{\sigma} \tilde{\xi}}\left(\alpha_{n}, r\right)\right.$ for each $r$-SVNSO set $\alpha_{n} \in \zeta^{\tilde{\mathcal{G}}}$.

Proof. (1) $\Rightarrow(2)$ : Let $x_{S, t, K} q f^{-1}\left(\alpha_{n}\right)$ and $\alpha_{n}$ is $r$-SVNRO set in $\tilde{\mathcal{G}}$. Then $\alpha_{n} \in Q_{\phi \tilde{\rho} \tilde{\sigma} \tilde{S}}\left(f\left(x_{s, t, k}\right), r\right)$.

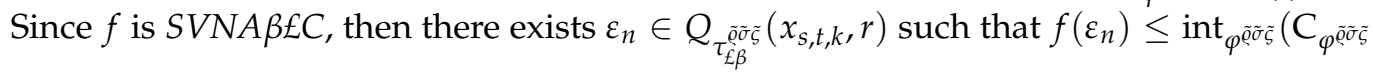
$\left.\left(\alpha_{n}, r\right), r\right)$. So,

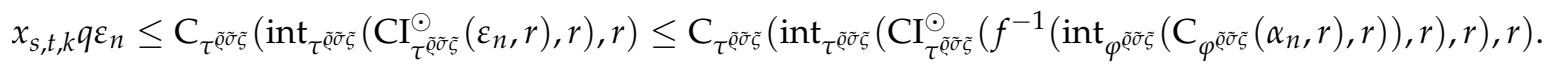

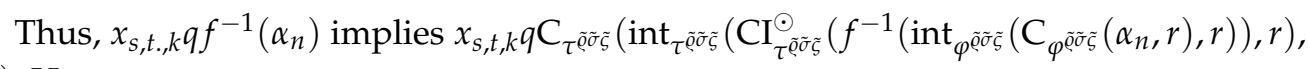
$r), r)$, Hence,

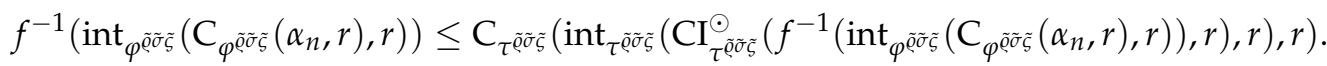

Therefore $f^{-1}\left(\operatorname{int}_{\varphi^{\tilde{\rho} \tilde{\sigma} \tilde{\xi}}}\left(\mathrm{C}_{\varphi_{\rho \tilde{Q} \tilde{\sigma} \tilde{\xi}}}\left(\alpha_{n}, r\right), r\right)\right)$ is an $r-S V N £ \beta O$ set in $\tilde{\mathcal{F}}$.

$(2) \Rightarrow(3)$ : Let $\varphi^{\tilde{\rho}}\left(\left[\alpha_{n}\right]^{c}\right) \geq r, \varphi^{\tilde{\sigma}}\left(\left[\alpha_{n}\right]^{c}\right) \leq 1-r, \varphi^{\tilde{S}}\left(\left[\alpha_{n}\right]^{c}\right) \leq 1-r$. Then, by (2),

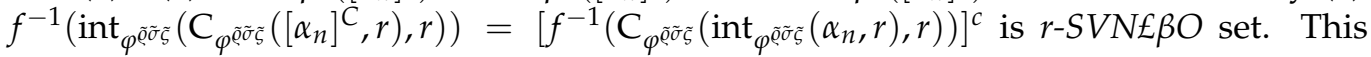

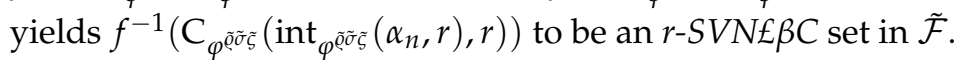

$(3) \Rightarrow(4)$ : Let $\alpha_{n}$ be an $r$-SVNRO set in $\tilde{\mathcal{G}}$. Then, $\varphi^{\tilde{\varphi}}\left(\alpha_{n}\right) \geq r, \varphi^{\tilde{\sigma}}\left(\alpha_{n}\right) \leq 1-r, \varphi^{\tilde{\zeta}}\left(\alpha_{n}\right) \leq$

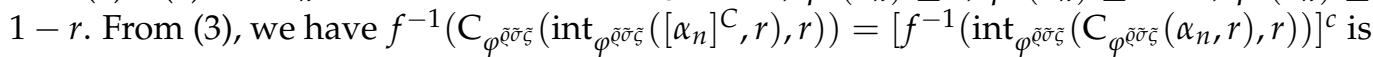
$r-S V N £ \beta C$ set. Hence, $f^{-1}\left(\alpha_{n}\right)$ is $r-S V N £ \beta O$ set in $\tilde{\mathcal{F}}$. 
$(4) \Rightarrow(5)$ : It is easily proved from (4) and the fact that $f^{-1}\left(\left[\alpha_{n}\right]^{c}\right)=\left[f^{-1}\left(\alpha_{n}\right)\right]^{c}$.

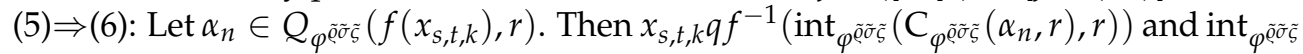

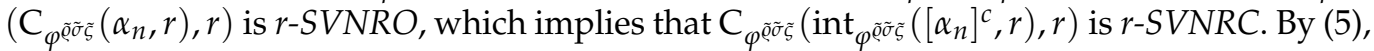

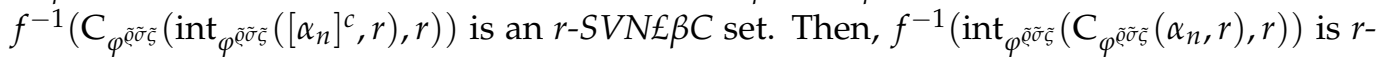

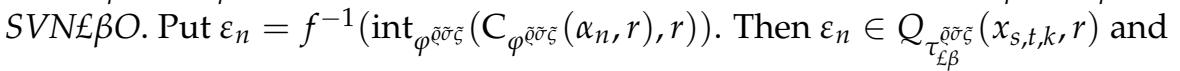

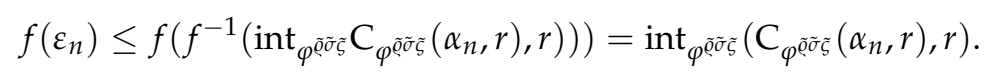

Since $\varphi^{\tilde{\rho}}\left(\alpha_{n}\right) \geq r, \varphi^{\tilde{\sigma}}\left(\alpha_{n}\right) \leq 1-r, \varphi^{\tilde{S}}\left(\alpha_{n}\right) \leq 1-r$ and by Lemma 1 , we have

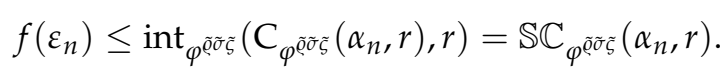

$(6) \Rightarrow(7)$ : Let $x_{S, t, K} q f^{-1}\left(\alpha_{n}\right)$ and $\varphi^{\tilde{\rho}}\left(\alpha_{n}\right) \geq r, \varphi^{\tilde{\sigma}}\left(\alpha_{n}\right) \leq 1-r, \varphi^{\tilde{S}}\left(\alpha_{n}\right) \leq 1-r$. Then,

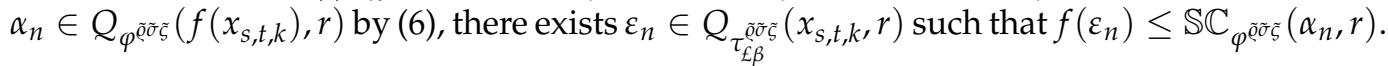
Thus, $\mathcal{B} \leq f^{-1}\left(\mathbb{S C}_{\varphi} \tilde{\tilde{o} \tilde{\sigma} \tilde{\zeta}}\left(\alpha_{n}, r\right)\right)$. Since $\varepsilon_{n}$ is $r$-SVN£ $\beta O$ set,

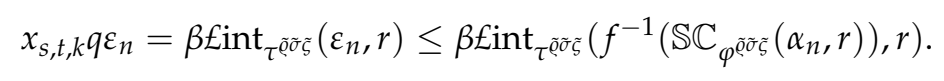

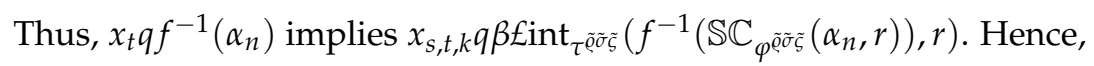

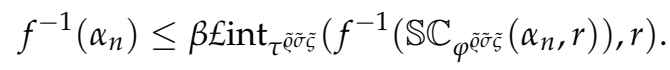

(7) $\Rightarrow(8)$ : Let $\varphi^{\tilde{\varrho}}\left(\left[\alpha_{n}\right]^{c}\right) \geq r, \varphi^{\tilde{\sigma}}\left(\left[\alpha_{n}\right]^{c}\right) \leq 1-r, \varphi^{\tilde{S}}\left(\left[\alpha_{n}\right]^{c}\right) \leq 1-r$. Then by (7),

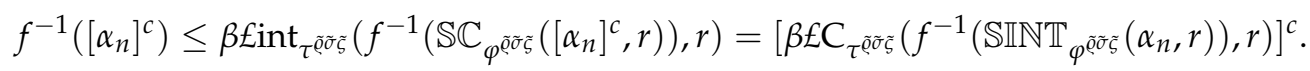

Then, $\beta \notin \mathrm{C}_{\tau \tilde{\varphi} \tilde{\sigma} \tilde{\sigma}}\left(f^{-1}\left(\operatorname{SINTT}_{\varphi \tilde{\sigma} \tilde{\sigma} \tilde{\zeta}}\left(\alpha_{n}, r\right)\right), r\right) \leq f^{-1}\left(\alpha_{n}\right)$.

$(8) \Rightarrow(9)$ : Since $\varphi^{\tilde{\varrho}}\left(\left[\alpha_{n}\right]^{c}\right) \geq r, \varphi^{\tilde{\sigma}}\left(\left[\alpha_{n}\right]^{c}\right) \leq 1-r, \varphi^{\tilde{S}}\left(\left[\alpha_{n}\right]^{c}\right) \leq 1-r$, by (8) and Lemma 1, we have

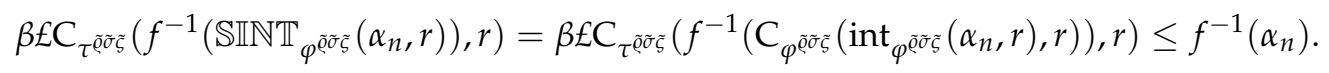

$(9) \Rightarrow(10)$ : Let $\alpha_{n}$ be an $r-S V N \beta O$ set in $\mathcal{G}$ Then by Lemma 2 ,

$$
\mathrm{C}_{\varphi^{\tilde{Q} \tilde{\sigma} \tilde{\zeta}}}\left(\alpha_{n}, r\right)=\mathrm{C}_{\varphi^{\tilde{Q} \tilde{\sigma} \tilde{\zeta}}}\left(\operatorname{int}_{\varphi^{\tilde{\sigma} \tilde{\sigma} \tilde{G}}}\left(\left(\mathrm{C}_{\varphi^{\tilde{Q} \tilde{\sigma} \tilde{\zeta}}}\left(\alpha_{n}, r\right), r\right), r\right)\right.
$$

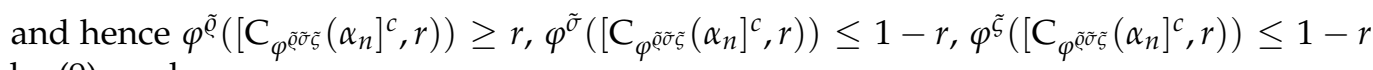
by (9), we have

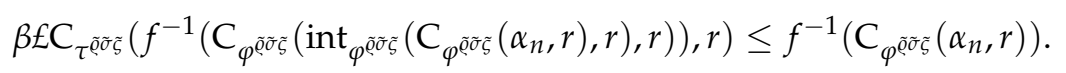

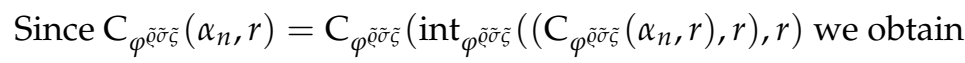

$$
\beta \notin \mathrm{C}_{\tau} \tilde{\partial \tilde{\sigma} \tilde{\zeta}}\left(f^{-1}\left(\alpha_{n}\right), r\right) \leq f^{-1}\left(\mathrm{C}_{\varphi \tilde{\sigma} \tilde{\sigma} \tilde{S}}\left(\alpha_{n}, r\right) .\right.
$$

$(10) \Rightarrow(11)$ : It is easily proved from Definition 9 .

$(11) \Rightarrow(1)$ : Obvious.

Theorem 8. Let $f:\left(\tilde{\mathcal{F}}, \tau^{\tilde{\rho} \tilde{\sigma} \tilde{S}}, £^{\tilde{\sigma} \tilde{\sigma} \tilde{S}}\right) \rightarrow\left(\tilde{\mathcal{G}}, \varphi^{\tilde{\rho} \tilde{\sigma} \tilde{S}}\right)$ be a mapping for each $\alpha_{n} \in \zeta^{\tilde{\mathcal{G}}}, \varepsilon_{n} \in \zeta^{\tilde{\mathcal{F}}}$ and $r \in \zeta_{0}$. Then the following statements are equivalent:

(1) $f$ is $S V N A B E C$,

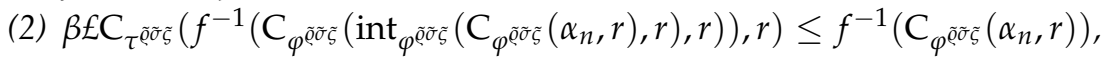




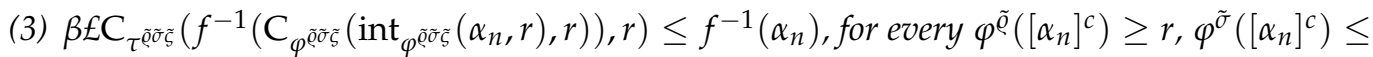
$1-r, \varphi^{\tilde{S}}\left(\left[\alpha_{n}\right]^{c}\right) \leq 1-r$,

(4) $\beta \notin \mathrm{C}_{\tau} \tilde{\tilde{\sigma} \tilde{\sigma} \tilde{\zeta}}\left(f^{-1}\left(\mathrm{C}_{\varphi \tilde{\rho} \tilde{\sigma} \tilde{G}}\left(\alpha_{n}, r\right)\right), r\right) \leq f^{-1}\left(\mathrm{C}_{\varphi^{\tilde{\sigma} \tilde{\sigma} \tilde{\zeta}}}\left(\alpha_{n}, r\right)\right)$, for every $\varphi^{\tilde{\varrho}}\left(\alpha_{n}\right) \geq r, \varphi^{\tilde{\sigma}}\left(\alpha_{n}\right) \leq 1-r$, $\left.\varphi^{\tilde{S}}\left(\alpha_{n}\right]\right) \leq 1-r$

(5) $f^{-1}\left(\alpha_{n}\right) \leq \beta \operatorname{Eint}_{\tau^{\tilde{\rho} \tilde{\sigma} \tilde{\zeta}}}\left(f^{-1}\left(\mathbb{S C}_{\varphi^{\tilde{Q} \tilde{\sigma} \tilde{\xi}}}\left(\alpha_{n}, r\right)\right), r\right)$, for every $\varphi^{\tilde{\rho}}\left(\alpha_{n}\right) \geq r, \varphi^{\tilde{\sigma}}\left(\alpha_{n}\right) \leq 1-r$, $\left.\varphi^{\tilde{S}}\left(\alpha_{n}\right]\right) \leq 1-r$

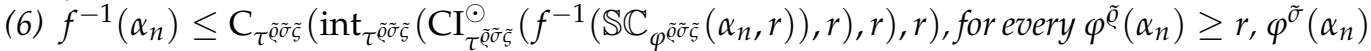
$\leq 1-r, \varphi^{\tilde{S}}\left(\alpha_{n}\right) \leq 1-r$

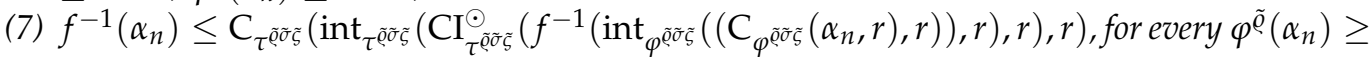
$r, \varphi^{\tilde{\sigma}}\left(\alpha_{n}\right) \leq 1-r, \varphi^{\tilde{S}}\left(\alpha_{n}\right) \leq 1-r$.

Proof. (1) $\Rightarrow(2)$ : Let $\alpha_{n} \in \zeta^{\tilde{\mathcal{G}}}$ and $x_{s, t, k} q\left[f^{-1}\left(\mathrm{C}_{\varphi \tilde{\sigma} \tilde{\sigma} \tilde{G}}\left(\alpha_{n}, r\right)\right)\right]^{c}$. Then, $\left.f\left(x_{s, t, k}\right) q\left[\mathrm{C}_{\varphi \tilde{\sigma} \tilde{\sigma} \tilde{S}}\left(\alpha_{n}, r\right)\right)\right]^{c}$ and since $\varphi^{\tilde{\rho}}\left(\left[\mathrm{C}_{\varphi \tilde{\rho} \tilde{\sigma} \tilde{S}}\left(\alpha_{n}, r\right)\right]^{c}\right) \geq r, \varphi^{\tilde{\sigma}}\left(\left[\mathrm{C}_{\varphi \tilde{\rho} \tilde{\sigma} \tilde{\zeta}}\left(\alpha_{n}, r\right)\right]^{c}\right) \leq 1-r, \varphi^{\tilde{S}}\left(\left[\mathrm{C}_{\varphi \tilde{\rho} \tilde{\sigma} \tilde{\sigma}}\left(\alpha_{n}, r\right)\right]^{c}\right) \leq 1-r$.

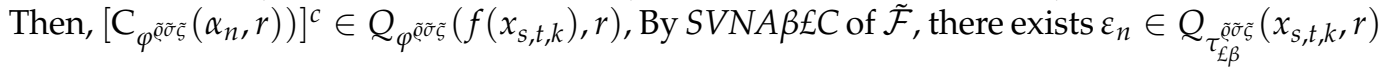
such that

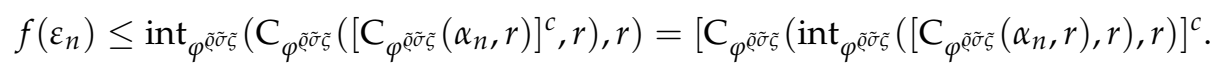
in $\tilde{\mathcal{F}}$

It implies that $\varepsilon_{n} \leq\left[f^{-1}\left(\mathrm{C}_{\varphi \tilde{\sigma} \tilde{\sigma} \tilde{S}}\left(\operatorname{int}_{\phi \tilde{\rho} \tilde{\sigma} \tilde{G}}\left(\left[\mathrm{C}_{\varphi \tilde{\varphi} \tilde{\sigma} \tilde{G}}\left(\alpha_{n}, r\right), r\right), r\right)\right)\right]^{c}\right.$. Since $\varepsilon_{n}$ is $S V N A \beta £ O$ set

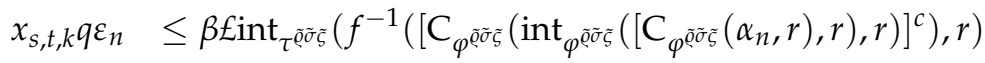

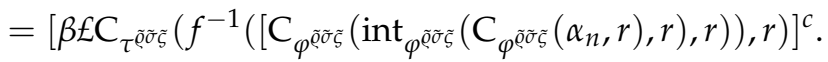

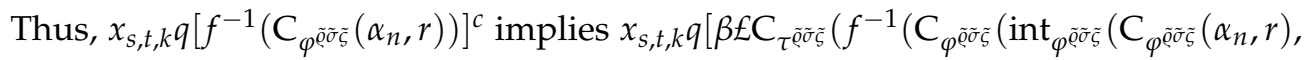
$r), r)), r)]^{c}$. Hence,

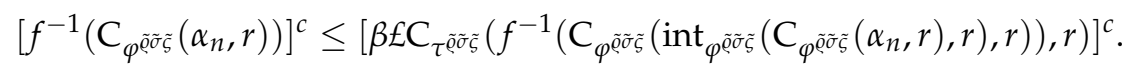

Thus,

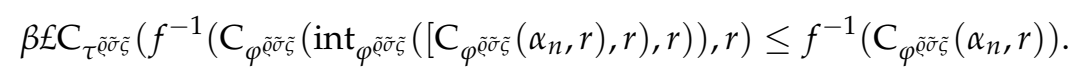

(2) $\Rightarrow(3)$ : It is trivial.

$(3) \Rightarrow(4)$ : Since $\varphi^{\tilde{\varrho}}\left(\alpha_{n}\right) \geq r, \varphi^{\tilde{\sigma}}\left(\alpha_{n}\right) \leq 1-r, \varphi^{\tilde{S}}\left(\alpha_{n}\right) \leq 1-r$, we have $\mathrm{C}_{\varphi^{\tilde{\sigma} \tilde{\sigma}} \tilde{\zeta}}\left(\alpha_{n}, r\right)=$

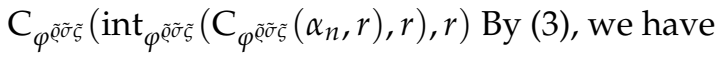

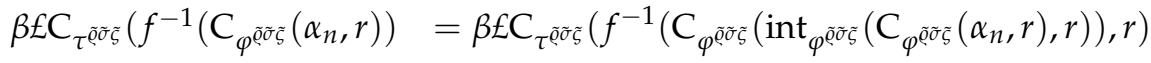

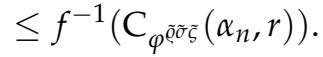

$(4) \Rightarrow(5)$ : Since $\varphi^{\tilde{\rho}}\left(\alpha_{n}\right) \geq r, \varphi^{\tilde{\sigma}}\left(\alpha_{n}\right) \leq 1-r, \varphi^{\tilde{S}}\left(\alpha_{n}\right) \leq 1-r$, we have $\varphi^{\tilde{\rho}}\left(\left[\mathrm{C}_{\varphi} \tilde{\varphi} \tilde{\sigma} \tilde{\zeta}\left(\alpha_{n}, r\right)\right]^{c}\right)$ $\geq r, \varphi^{\tilde{\sigma}}\left(\left[\mathrm{C}_{\varphi^{\tilde{\sigma} \tilde{\sigma} \tilde{S}}}\left(\alpha_{n}, r\right)\right]^{c}\right) \leq 1-r, \varphi^{\tilde{S}}\left(\left[\mathrm{C}_{\varphi^{\tilde{\sigma} \tilde{\sigma} \tilde{\sigma}}}\left(\alpha_{n}, r\right)\right]^{c}\right) \leq 1-r$. and by Lemma 1, we have $\mathbb{S C}_{\varphi^{\tilde{\rho} \tilde{\sigma} \tilde{\xi}}\left(\alpha_{n}, r\right)=\operatorname{int}_{\varphi} \tilde{\sigma} \tilde{\sigma} \tilde{\zeta}}\left(\mathrm{C}_{\varphi^{\tilde{\rho} \tilde{\sigma} \tilde{\xi}}}\left(\alpha_{n}, r\right), r\right)$. From (4), we have

$$
\begin{aligned}
& {\left[\beta \operatorname{int}_{\tau^{\tilde{\rho} \tilde{\sigma} \tilde{\zeta}}}\left(f^{-1}\left(\operatorname{int}_{\varphi^{\tilde{\sigma} \tilde{\sigma} \tilde{\zeta}}}\left(\mathrm{C}_{\varphi^{\tilde{\sigma} \tilde{\sigma} \tilde{\zeta}}}\left(\alpha_{n}, r\right), r\right)\right), r\right)\right]^{c}=\beta £ \mathrm{C}_{\tau^{\tilde{\rho} \tilde{\sigma} \tilde{\zeta}}}\left(f^{-1}\left(\mathrm{C}_{\varphi^{\tilde{\rho} \tilde{\sigma} \tilde{\zeta}}}\left(\left[\mathrm{C}_{\varphi^{\tilde{\rho} \tilde{\sigma} \tilde{\zeta}}}\left(\alpha_{n}, r\right)\right]^{n}, r\right)\right), r\right)}
\end{aligned}
$$

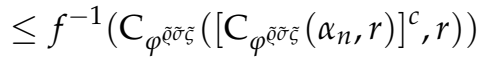

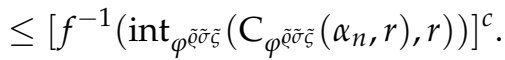

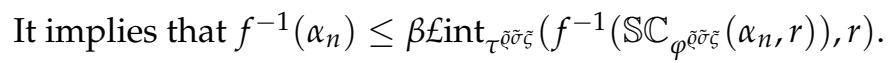


(5) $\Rightarrow(6)$ : Let $\varphi^{\tilde{\rho}}\left(\alpha_{n}\right) \geq r, \varphi^{\tilde{\sigma}}\left(\alpha_{n}\right) \leq 1-r, \varphi^{\tilde{S}}\left(\alpha_{n}\right) \leq 1-r$. Then by (5), we have

$$
\begin{aligned}
& f^{-1}\left(\alpha_{n}\right) \leq \beta \operatorname{sint}_{\tau} \tilde{\tilde{\sigma} \tilde{\sigma} \tilde{\zeta}}\left(f^{-1}\left(\mathbb{S C}_{\varphi \tilde{Q} \tilde{\sigma} \tilde{\sigma}}\left(\alpha_{n}, r\right)\right), r\right)
\end{aligned}
$$

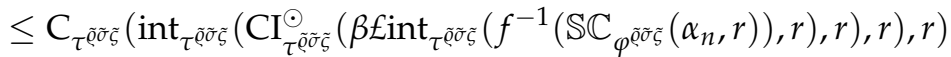

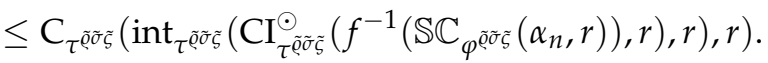

$(6) \Rightarrow(7)$ : It is easily proved from Lemma 1.

$(7) \Rightarrow(1)$ : Let $\alpha_{n} \in Q_{\varphi^{\tilde{O} \tilde{\sigma} \tilde{\sigma}}}\left(f\left(x_{s, t, k}\right), r\right)$, then $x_{s, t, k} q f^{-1}\left(\alpha_{n}\right)$ and $\varphi^{\tilde{Q}}\left(\alpha_{n}\right) \geq r, \varphi^{\tilde{\sigma}}\left(\alpha_{n}\right) \leq 1-$

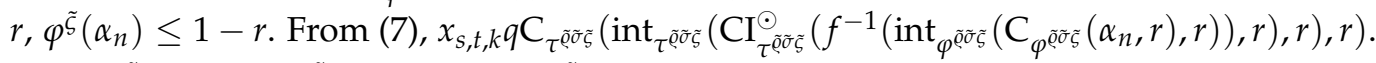

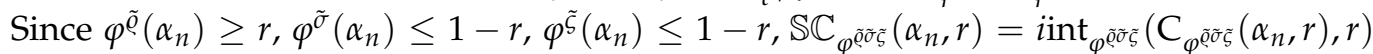

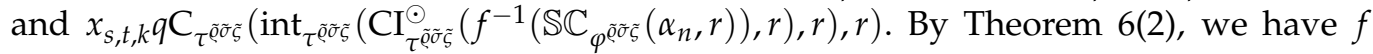
is $S V N A \beta E C$.

Definition 19. Let $f:\left(\tilde{\mathcal{F}}, \tau^{\tilde{\rho} \tilde{\sigma} \tilde{\zeta}}, £^{\tilde{\rho} \tilde{\sigma} \tilde{S}}\right) \rightarrow\left(\tilde{\mathcal{G}}, \varphi^{\tilde{\rho} \tilde{\sigma} \tilde{s}}\right)$ be a mapping. Then,

(1) $f$ is called single-valued neutrosophic faintly $\beta E$-continuous ( $S V N F \beta \notin C$, for short) iff for every $\alpha_{n} \in Q_{\varphi \tilde{\sigma} \tilde{\sigma} \tilde{\xi}}\left(f\left(x_{s, t, k}\right), r\right)$, there exists $\varepsilon_{n} \in Q_{\tau_{\tilde{\varepsilon} \beta}^{\tilde{\tilde{\sigma}} \tilde{\xi}}}\left(x_{s, t, k}, r\right)$ such that $f\left(\varepsilon_{n}\right) \leq \alpha_{n}$,

(2) $f$ is called single-valued neutrosophic weakly $\beta E$-continuous ( $S V N W \beta \notin C$, for short) iff for every

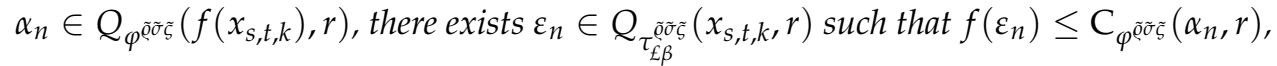

(3) $f$ is called single-valued neutrosophic $\beta \notin$-continuous ( $S V N \beta E C$, for short) iff $f^{-1}\left(\alpha_{n}\right)$ is $r$ $\operatorname{SVN} £ \beta O$, for every $\varphi^{\tilde{\rho}}\left(\alpha_{n}\right) \geq r, \varphi^{\tilde{\sigma}}\left(\alpha_{n}\right) \leq 1-r, \varphi^{\tilde{S}}\left(\alpha_{n}\right) \leq 1-r$.

Remark 3. From the above definition we obtain the following diagram:

$$
\begin{aligned}
S V N \beta E C & \Rightarrow S V N A \beta E C \\
\Downarrow & \\
S V N F \beta E C & \Leftarrow S V N W \beta E C
\end{aligned}
$$

Some supporting examples will be shown after the following two theorems.

Theorem 9. Let $f:\left(\tilde{\mathcal{F}}, \tau^{\tilde{\rho} \tilde{\sigma} \tilde{S}}, £^{\tilde{\sigma} \tilde{\sigma} \tilde{S}}\right) \rightarrow\left(\tilde{\mathcal{G}}, \varphi^{\tilde{Q} \tilde{\sigma} \tilde{S}}\right)$ be a mapping. Then the following statements are equivalent:

(1) $f$ is $S V N W \beta E C$,

(2) $f\left(\beta £ C_{\tau \tilde{\sigma} \tilde{\sigma} \tilde{\xi}}\left(\alpha_{n}, r\right)\right) \leq \Theta_{\varphi \bar{o} \tilde{\sigma} \tilde{\zeta}}\left(f\left(\alpha_{n}\right), r\right)$, for each $\alpha_{n} \in \zeta^{\tilde{\mathcal{F}}}, r \in \zeta_{0}$,

(3) $\beta \notin C_{\tau} \tilde{\tilde{\rho} \tilde{\sigma} \tilde{G}}\left(f^{-1}\left(\varepsilon_{n}\right), r\right) \leq f^{-1}\left(\Theta_{\varphi \tilde{o} \tilde{\sigma} \tilde{\xi}}\left(\varepsilon_{n}, r\right)\right)$, for each $\varepsilon_{n} \in \zeta^{\tilde{\mathcal{G}}}, r \in \zeta_{0}$,

(4) $f^{-1}\left(\varepsilon_{n}\right)$ is $r$-SVN $\beta \notin C$ set in $\tilde{\mathcal{F}}$ for each $r$ - $\theta$-closed set,

(5) $f^{-1}\left(\varepsilon_{n}\right)$ isr-SVN $\beta £ O$ set in $\tilde{\mathcal{F}}$ for each $r-\theta$-open set.

Proof. (1) $\Rightarrow(2)$ Suppose there exists $\alpha_{n} \in \zeta^{\tilde{\mathcal{F}}}$ and $r \in \zeta_{0}$ such that $f\left(\beta \notin \mathrm{C}_{\tau \tilde{\varphi} \tilde{\sigma} \tilde{\zeta}}\left(\alpha_{n}, r\right)\right) \not \leq$ $\Theta_{\varphi \rho \tilde{\sigma} \tilde{\xi}}\left(f\left(\alpha_{n}\right), r\right)$. Then there exists $v \in \tilde{\mathcal{G}}$ and $s, t, k \in \zeta_{0}$ such that

$$
\begin{aligned}
& \varphi_{f\left(\beta \notin \mathrm{C}_{\tau \tilde{\tau}}\left(\alpha_{n}, r\right)\right)}^{\tilde{\rho}}(v)>s>\varphi_{\Theta_{\phi^{\tilde{\rho}}}^{\tilde{\rho}}\left(f\left(\alpha_{n}\right), r\right)}^{\tilde{\rho}}(v) \\
& \varphi_{f\left(\beta \notin \mathrm{C}_{\tau \tilde{\tau}} \tilde{\sigma}\left(\alpha_{n}, r\right)\right)}^{\tilde{\sigma}}(v) \leq t \leq \varphi_{\Theta_{\varphi^{\tilde{\sigma}}}^{\tilde{\sigma}}}\left(f\left(\alpha_{n}\right), r\right) \\
& \varphi_{f\left(\beta \notin \mathrm{C}_{\tau \tilde{\xi}}\left(\alpha_{n}, r\right)\right)}^{\tilde{\zeta}}(v) \leq k \leq \varphi_{\Theta_{\phi^{\tilde{\xi}}}\left(f\left(\alpha_{n}\right), r\right)}^{\tilde{\zeta}}(v)
\end{aligned}
$$


If $f^{-1}(\{v\})=\varnothing$, provides a contradiction that $f\left(\beta £ C_{\tau} \tilde{\tilde{\sigma} \tilde{\sigma} \tilde{\xi}}\left(\alpha_{n}, r\right)\right)=0$. If $f^{-1}(\{v\}) \neq$ $\varnothing$, there exists $\omega \in f^{-1}(\{v\})$ such that

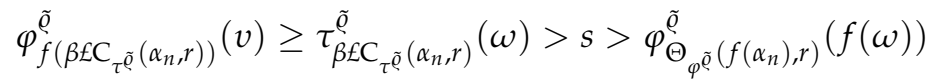

$$
\begin{aligned}
& \varphi_{f\left(\beta \notin C_{\tau^{\tilde{\sigma}}}\left(\alpha_{n}, r\right)\right)}^{\tilde{\sigma}}(v) \leq \tau_{\beta \notin C_{\tau^{\tilde{\sigma}}}^{\tilde{\sigma}}\left(\alpha_{n}, r\right)}^{\tilde{\sigma}}(\omega) \leq t \leq \varphi_{\Theta_{\varphi^{\tilde{\sigma}}}^{\tilde{\sigma}}\left(f\left(\alpha_{n}\right), r\right)}^{\tilde{\sigma}}(f(\omega)) \\
& \varphi_{f\left(\beta \notin \mathrm{C}_{\tau \tilde{\zeta}}\left(\alpha_{n}, r\right)\right)}^{\tilde{\zeta}}(v) \leq \tau_{\beta \notin \mathrm{C}_{\tau} \tilde{\zeta}\left(\alpha_{n}, r\right)}^{\tilde{\zeta}}(\omega) \leq k \leq \varphi_{\Theta_{\varphi \tilde{\zeta}}^{\tilde{\zeta}}\left(f\left(\alpha_{n}\right), r\right)}(f(\omega)) .
\end{aligned}
$$

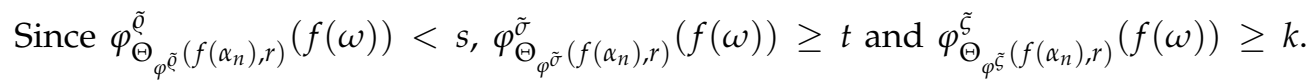
Then, $f(x)_{s, t, k}$ is not $r$ - $\theta$-cluster point of $f\left(\alpha_{n}\right)$, there exists $\varepsilon_{n} \in Q_{\varphi(\tilde{\sigma} \tilde{\sigma} \tilde{\xi}}\left(f\left(x_{s, t, k}\right), r\right)$ such

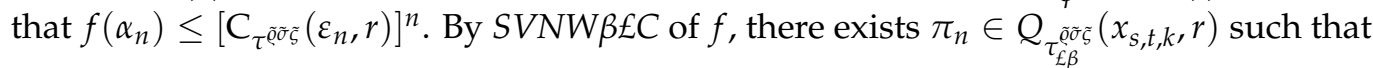
$f\left(\pi_{n}\right) \leq \mathrm{C}_{\varphi \bar{o} \tilde{\sigma} \tilde{\zeta}}\left(\varepsilon_{n}, r\right)$. Thus, $f\left(\alpha_{n}\right) \leq\left[f\left(\pi_{n}\right)\right]^{c}$ implies $\alpha_{n} \leq\left[\pi_{n}\right]^{c}$. Hence

$$
\begin{aligned}
& \tau_{\beta \notin C_{\tau} \tilde{\rho}\left(\alpha_{n}, r\right)}^{\tilde{\rho}}(\omega) \leq \tau_{\left[\pi_{n}\right]^{c}}^{\tilde{\rho}}(\omega)<s, \\
& \tau_{\beta \notin C_{\tau^{\tilde{\sigma}}}^{\tilde{\sigma}}\left(\alpha_{n}, r\right)}^{\tilde{\sigma}}(\omega) \geq \tau_{\left[\pi_{n}\right]^{\tilde{\sigma}}}^{\tilde{\sigma}}(\omega) \geq t, \\
& \tau_{\beta \notin C_{\tau} \tilde{\zeta}\left(\alpha_{n}, r\right)}^{\tilde{\zeta}}(\omega) \geq \tau_{\left[\pi_{n}\right]^{\tilde{\zeta}}}(\omega) \geq t .
\end{aligned}
$$

It is a contradiction for Equation (2).

$(2) \Rightarrow(3),(3) \Rightarrow(4)$ and $(4) \Rightarrow(5)$ : are obvious.

$(5) \Rightarrow(1)$ : Let $\alpha_{n} \in Q_{\varphi(\tilde{\sigma} \tilde{\sigma}}\left(f\left(x_{s, t, k}\right), r\right)$. Then $x_{s, t, k} q f^{-1}\left(\alpha_{n}\right)$ and $\alpha_{n}$ is $r$ - $\theta$-open set. By (5), we have $f^{-1}\left(\alpha_{n}\right)$ is $r$-SVN $\beta £ O$ set in $\tilde{\mathcal{F}}$. Since $x_{s, t, k} q f^{-1}\left(\alpha_{n}\right)$ we obtain $f^{-1}\left(\alpha_{n}\right) \in$

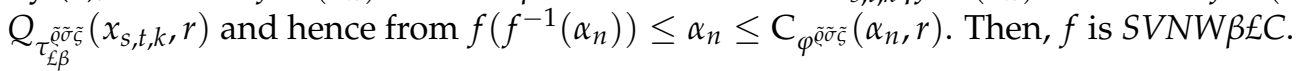

Theorem 10. A mapping $f:\left(\tilde{\mathcal{F}}, \tau^{\tilde{Q} \tilde{\sigma} \tilde{S}}, £^{\tilde{Q} \tilde{\sigma} \tilde{S}}\right) \rightarrow\left(\tilde{\mathcal{G}}, \varphi^{\tilde{\tilde{\sigma}} \tilde{S}}\right)$ is SVNFß£C iff for each $r$ - $\theta$-closed set $\alpha_{n} \in \zeta^{\tilde{\mathcal{G}}}, f^{-1}\left(\alpha_{n}\right)$ is $r-S V N \beta E C$.

Proof. Obvious.

Example 2. Let $\mathcal{F}=\{a, b\}$. Define $\varepsilon_{n}, \pi_{n} \in \zeta^{\mathcal{F}}$ as follows:

$$
\varepsilon_{n}=\langle(0.3,0.3),(0.7,0.7),(0.3,0.3)\rangle ; \pi_{n}=\langle(0.3,0.3),(0.3,0.3),(0.3,0.3)\rangle .
$$

We define the mapping $\tau^{\tilde{\rho} \tilde{\sigma} \tilde{\zeta}}, \varphi^{\tilde{\varphi} \tilde{\sigma} \tilde{\zeta}}, £^{\tilde{\rho} \tilde{\sigma} \tilde{\zeta}}: \zeta^{\mathcal{F}} \times \zeta_{0} \rightarrow \zeta^{\mathcal{F}}$ as follows:

$$
\begin{aligned}
& \tau^{\tilde{\rho}}\left(\alpha_{n}\right)=\left\{\begin{array}{ll}
1, & \text { if } \alpha_{n}=\tilde{1} \text { or } \tilde{0}, \\
\frac{2}{3}, & \text { if } \alpha_{n}=\varepsilon_{n} \text { or } \pi_{n} \\
0, & \text { otherwise, }
\end{array} \quad \varphi^{\tilde{\rho}}\left(\alpha_{n}\right)= \begin{cases}1, & \text { if } \alpha_{n}=\tilde{1} \text { or } \tilde{0}, \\
\frac{2}{3}, & \text { if } \alpha_{n}=\pi_{n} \\
0, & \text { otherwise, }\end{cases} \right. \\
& \tau^{\tilde{\sigma}}\left(\alpha_{n}\right)=\left\{\begin{array}{cc}
0, & \text { if } \alpha_{n}=\tilde{1} \text { or } \tilde{0}, \\
\frac{1}{3}, & \text { if } \alpha_{n}=\varepsilon_{n} \text { or } \pi_{n} \\
1, & \text { otherwise, }
\end{array} \quad \varphi^{\tilde{\sigma}}\left(\alpha_{n}\right)=\left\{\begin{array}{cc}
0, & \text { if } \alpha_{n}=\tilde{1} \text { or } \tilde{0}, \\
\frac{1}{3}, & \text { if } \alpha_{n}=\pi_{n} \\
0, & \text { otherwise, }
\end{array}\right.\right. \\
& \tau^{\tilde{S}}\left(\alpha_{n}\right)=\left\{\begin{array}{cc}
0, & \text { if } \alpha_{n}=\tilde{1} \text { or } \tilde{0}, \\
\frac{1}{3}, & \text { if } \alpha_{n}=\varepsilon_{n} \text { or } \pi_{n} \\
1, & \text { otherwise, }
\end{array} \quad \varphi^{\tilde{S}}\left(\alpha_{n}\right)=\left\{\begin{array}{cc}
0, & \text { if } \alpha_{n}=\tilde{1} \text { or } \tilde{0}, \\
\frac{1}{3}, & \text { if } \alpha_{n}=\pi_{n} \\
1, & \text { otherwise, }
\end{array}\right.\right.
\end{aligned}
$$




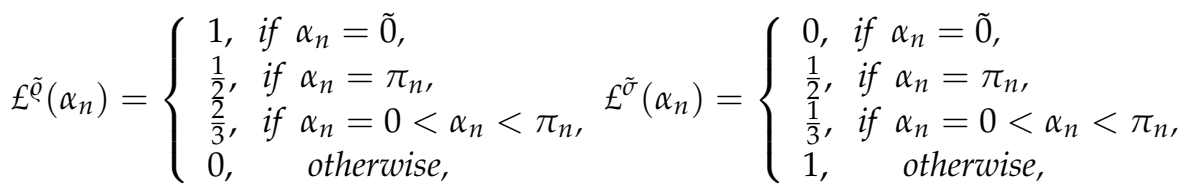

$$
\begin{aligned}
& £^{\tilde{S}}\left(\alpha_{n}\right)= \begin{cases}0, & \text { if } \alpha_{n}=\tilde{0} \\
\frac{1}{2}, & \text { if } \alpha_{n}=\pi_{n} \\
\frac{1}{3}, & \text { if } \alpha_{n}=0<\alpha_{n}<\pi_{n} \\
1, & \text { otherwise }\end{cases}
\end{aligned}
$$

From Theorems 4 and 5 , we obtain $\beta \mathcal{I} C_{\tau}, T_{\tau}: I^{X} \times I_{0} \rightarrow I^{X}$ as follows:

$$
\begin{aligned}
& \Theta^{\tau \tilde{\tau} \tilde{\sigma} \tilde{s}}\left(\alpha_{n}, r\right)= \begin{cases}\tilde{0}, & \text { if } \alpha_{n}=\tilde{0}, \\
\varepsilon_{n}, & \text { if } \tilde{0} \neq \alpha_{n} \leq \pi_{n}, \quad 0<r \leq \frac{2}{3}, \\
\tilde{1}, & \text { otherwise, }\end{cases} \\
& \beta \notin \mathrm{C}_{\tau \tilde{\varrho} \tilde{\sigma} \tilde{\mathcal{S}}}\left(\alpha_{n}, r\right)=\left\{\begin{array}{l}
\tilde{0}, \text { if } \mathcal{B}=\underline{0}, \\
\pi_{n}, \text { if } \tilde{0} \neq \alpha_{n} \leq \pi_{n}, \quad 0<r \leq \frac{2}{3}, \\
\tilde{1}, \quad \text { otherwise, }
\end{array}\right.
\end{aligned}
$$

By Theorem 9(2), the identity mapping id ${ }_{X}::\left(\tilde{\mathcal{F}}, \tau^{\tilde{\rho} \tilde{\sigma} \tilde{S}}, £^{\tilde{Q} \tilde{\sigma} \tilde{S}}\right) \rightarrow\left(\tilde{\mathcal{G}}, \varphi^{\tilde{\rho} \tilde{\sigma} \tilde{S}}\right)$ is SVNW $\beta £ C$ but is not SVNAß£C, because by Theorem 8(5), for each $\varphi^{\tilde{\rho}}\left(\pi_{n}\right) \geq \frac{2}{3}, \varphi^{\tilde{\sigma}}\left(\pi_{n}\right) \leq \frac{1}{3}, \varphi^{\tilde{S}}\left(\pi_{n}\right) \leq \frac{1}{3}$ and $\varphi^{\tilde{S}}\left(\pi_{n}\right) \leq \frac{1}{3}$

$$
\tilde{1}=\beta \notin \mathrm{C}_{\tau \tilde{\vartheta} \tilde{\sigma} \tilde{\zeta}}\left(\mathrm{C}_{\tau \tilde{\varphi} \tilde{\sigma} \tilde{\zeta}}\left(\pi_{n}, \frac{2}{3}\right)\right) \not \leq \mathrm{C}_{\varphi \tilde{\sigma} \tilde{\sigma} \tilde{\zeta}}\left(\pi_{n}, \frac{2}{3}\right)=\varepsilon_{n} .
$$

\section{Single-Valued Neutrosophic Approximation Space}

In this section, and for symmetrical purposes, we establish the definition of the single-valued neutrosophic upper, single-valued neutrosophic lower and single-valued neutrosophic boundary sets of a rough single-valued neutrosophic set $\alpha_{n}$ in a singlevalued neutrosophic approximation space $(\tilde{\mathcal{F}}, \delta)$. Based on $\alpha_{n}$ and $\delta$, we introduce the single-valued neutrosophic approximation interior operator int $t_{\alpha_{n}}^{\delta}$ and the single-valued neutrosophic approximation closure operator $\mathrm{Cl}_{\alpha_{n}}^{\delta}$.

Definition 20. Assume that an SVNR $\delta$ is defined so that $\tilde{\varrho}_{\delta}(\omega, \omega)=1, \tilde{\sigma}_{\delta}(\omega, \omega)$ $=0, \tilde{\zeta}_{\delta}(\omega, \omega)=0$ for every $\omega \in \tilde{\mathcal{F}}, \tilde{\varrho}_{\delta}(\omega, v)=\tilde{\varrho}_{\alpha_{n}}(v, \omega), \tilde{\sigma}_{\delta}(\omega, v)=\tilde{\sigma}_{\delta}(v, \omega), \tilde{\zeta}_{\delta}(\omega, v)=$ $\tilde{\zeta}_{\alpha_{n}}(\nu, \omega)$ for every $\omega, v \in \tilde{\mathcal{F}}$ and $\tilde{\varrho}_{\delta}(\omega, v) \geq\left(\tilde{\varrho}_{\delta}(\omega, \mu) \wedge \tilde{\varrho}_{\delta}(\mu, v)\right), \tilde{\sigma}_{\delta}(\omega, v) \leq\left(\tilde{\sigma}_{\delta}(\omega, \mu) \vee\right.$ $\left.\tilde{\sigma}_{\delta}(\mu, v)\right)$ and $\tilde{\zeta}_{\delta}(\omega, v) \leq\left(\tilde{\zeta}_{\delta}(\omega, \mu) \vee \tilde{\zeta}_{\delta}(\mu, v)\right)$ for every $\omega, v, \mu \in \tilde{\mathcal{F}}$. That is, $\delta$ is a single-valued neutrosophic equivalence relation on $\tilde{\mathcal{F}}$. Then $(\tilde{\mathcal{F}}, \delta)$ is called a single-valued neutrosophic approximation space based on the single-valued neutrosophic equivalence relation (briefly, SVN-equivalence relation) $\delta$ on $\tilde{\mathcal{F}}$.

Definition 21. For each $\omega \in \tilde{\mathcal{F}}$, define a single-valued neutrosophic coset $[\omega]: \tilde{\mathcal{F}} \Rightarrow[0,1]$ by:

$$
\tilde{\varrho}_{[\omega]}(v)=\tilde{\sigma}_{\delta}(\omega, v), \quad \tilde{\sigma}_{[\omega]}(v)=\tilde{\sigma}_{\delta}(\omega, v), \quad \tilde{\zeta}_{[\omega]}(v)=\tilde{\zeta}_{\delta}(\omega, v) \quad \forall v \in . \tilde{\mathcal{F}} .
$$

All elements $v \in \tilde{\mathcal{F}}$ with $\operatorname{SVNR}$ value $\tilde{\varrho}_{\delta}(\omega, v)>0, \tilde{\sigma}_{\delta}(\omega, v) \leq 1, \tilde{\varsigma}_{\delta}(\omega, v) \leq 1$ are elements having a membership value in the single-valued neutrosophic coset $[\omega]$, and any element $v \in . \tilde{\mathcal{F}}$ with $\tilde{\varrho}_{\delta}(\omega, \omega)=1, \tilde{\sigma}_{\delta}(\omega, \omega)=0, \tilde{\zeta}_{\delta}(\omega, \omega)=0$ is not included in the single-valued neutrosophic coset $[\omega]$. Any single-valued neutrosophic coset $[\omega]$ surely include the element $\omega \in \tilde{\mathcal{F}}$, and consequently

$$
\tilde{\varrho}_{\bigvee_{\mu \in \tilde{\mathcal{F}}}}([\omega](\mu))=1, \quad \tilde{\sigma}_{\Lambda_{\mu \in \tilde{\mathcal{F}}}}([\omega](\mu))=0, \quad \tilde{\varsigma}_{\Lambda_{\mu \in \tilde{\mathcal{F}}}}([\omega](\mu))=0, \quad \forall \omega \in \tilde{\mathcal{F}}
$$




\section{Further,}

$$
\tilde{\varrho}_{\bigvee_{\mu \in \tilde{\mathcal{F}}}}([\omega](v))=1, \quad \tilde{\sigma}_{\bigwedge_{\mu \in \tilde{\mathcal{F}}}}([\omega](v))=0, \quad \tilde{\mathcal{S}}_{\bigwedge_{\mu \in \tilde{\mathcal{F}}}}([\omega](v))=0, \quad \forall v \in \tilde{\mathcal{F}},
$$

such that $\bigvee_{v \in \tilde{\mathcal{F}}}([v])=\langle 0,1,1\rangle$. Clearly, if $\tilde{\varrho}_{\delta}(\omega, v)>0, \tilde{\sigma}_{\delta}(\omega, v) \leq 1, \tilde{\zeta}_{\delta}(\omega, v) \leq 1$, then the single-valued neutrosophic cosets $[\omega],[v]$ (as SVNSs) are containing the same elements of $\tilde{\mathcal{F}}$ with some non-zero membership values, and moreover, if $\tilde{\varrho}_{[v]}(\mu)=0, \quad \tilde{\sigma}_{[v]}(\mu)=1$ and $\tilde{\zeta}[\omega](\mu)=1$, then it must be that $\tilde{\varrho}_{[\omega]}(\mu)=0, \quad \tilde{\sigma}_{[\omega]}(\mu)=1$ and $\tilde{\zeta}_{[v]}(\mu)=1$ whenever $\tilde{\varrho}_{\delta}(\omega, v)>0, \tilde{\sigma}_{\delta}(\omega, v) \leq 1, \tilde{\zeta}_{\delta}(\omega, v) \leq 1$. That is, any two single-valued neutrosophic cosets are either two single-valued neutrosophic sets containing the same elements of $\tilde{\mathcal{F}}$ with some nonzero membership values or containing completely different elements of $\tilde{\mathcal{F}}$ with some non-zero membership values.

Definition 22. Let $\alpha_{n} \in \zeta^{\tilde{\mathcal{F}}}$ and $\delta$ be $S V N$-equivalence relation on $\tilde{\mathcal{F}}$ and the single-valued neutrosophic cosets. Then, the single-valued neutrosophic lower set (briefly, SVN-lower) $\left(\alpha_{n}\right)^{\delta}$, the single-valued neutrosophic upper set (briefly, SVN-upper) $\left(\alpha_{n}\right)_{\delta}$ and the single-valued neutrosophic boundary region set (briefly, $S V N$-boundary region) $\left(\alpha_{n}\right)^{B}$ are defined as follows: for $\omega \in \tilde{\mathcal{F}}$,

$$
\begin{aligned}
& \tilde{\varrho}_{\left(\alpha_{n}\right)_{\delta}}(\omega)=\tilde{\varrho}_{\alpha_{n}}(\omega) \vee \bigwedge_{\left(\alpha_{n}\right)^{c}(\mu)>0, \mu \neq \omega} \tilde{\mathcal{S}}_{[\omega]}(\mu), \quad \tilde{\sigma}_{\left(\alpha_{n}\right)_{\delta}}(\omega)=\tilde{\sigma}_{\alpha_{n}}(\omega) \wedge \bigvee_{\left(\alpha_{n}\right)^{c}(\mu)>0, \mu \neq \omega}\left(1-\tilde{\sigma}_{[\omega]}\right)(\mu) \\
& \tilde{\zeta}_{\left(\alpha_{n}\right)_{\delta}}(\omega)=\tilde{\zeta}_{\alpha_{n}}(\omega) \wedge \bigvee_{\left(\alpha_{n}\right)^{c}(\mu)>0, \mu \neq \omega} \tilde{\varrho}_{[\omega]}(\mu) \\
& \tilde{\varrho}_{\left(\alpha_{n}\right)^{\delta}}(\omega)=\tilde{\varrho}_{\alpha_{n}}(\omega) \wedge \bigvee_{\alpha_{n}(\mu)>0, \mu \neq \omega} \tilde{\varrho}_{[\omega]}(\mu), \quad \tilde{\sigma}_{\left(\alpha_{n}\right)^{\delta}}(\omega)=\tilde{\sigma}_{\alpha_{n}}(\omega) \vee \bigwedge_{\alpha_{n}(\mu)>0, \mu \neq \omega}\left(\tilde{\sigma}_{[\omega]}(\mu)\right. \\
& \tilde{S}_{\left(\alpha_{n}\right)^{\delta}}(\omega)=\tilde{\zeta}_{\alpha_{n}}(\omega) \vee \bigwedge_{\alpha_{n}(\mu)>0, \mu \neq \omega} \tilde{S}_{[\omega]}(\mu) \\
& \tilde{\varrho}_{\left(\alpha_{n}\right)^{B}}(\omega)=\tilde{\varrho}_{\left(\alpha_{n}\right)^{\delta}} \bar{\wedge} \tilde{\varrho}_{\left(\alpha_{n}\right)_{\delta}}= \begin{cases}\tilde{0}, & \text { if }\left(\alpha_{n}\right)^{\delta} \leq\left(\alpha_{n}\right)_{\delta}, \\
\left(\alpha_{n}\right)^{\delta} \wedge\left[\left(\alpha_{n}\right)_{\delta}\right]^{c}, & \text { otherwise, }\end{cases} \\
& \tilde{\sigma}_{\left(\alpha_{n}\right)^{B}}(\omega)=\tilde{\sigma}_{\left(\alpha_{n}\right)^{\delta}} \bar{\nabla} \tilde{\sigma}_{\left(\alpha_{n}\right)_{\delta}}= \begin{cases}\tilde{1}_{1}, & \text { if }\left(\alpha_{n}\right)^{\delta} \geq\left(\alpha_{n}\right)_{\delta}, \\
\left(\alpha_{n}\right)^{\delta} \vee\left[\left(\alpha_{n}\right)_{\delta}\right]^{c}, & \text { otherwise, }\end{cases} \\
& \tilde{\zeta}_{\left(\alpha_{n}\right)^{B}}(\omega)=\tilde{S}_{\left(\alpha_{n}\right)^{\delta}} \bar{\nabla} \tilde{S}_{\left(\alpha_{n}\right)_{\delta}}= \begin{cases}\tilde{1}_{,} & \text {if }\left(\alpha_{n}\right)^{\delta} \geq\left(\alpha_{n}\right)_{\delta}, \\
\left(\alpha_{n}\right)^{\delta} \vee\left[\left(\alpha_{n}\right)_{\delta}\right]^{c}, & \text { otherwise, }\end{cases}
\end{aligned}
$$

$\left(\alpha_{n}\right)^{\delta},\left(\alpha_{n}\right)_{\delta}$ and $\left(\alpha_{n}\right)^{B}$ are then called SVN-lower, SVN-upper and SVN-boundary region sets associated with the SVNS $\alpha_{n} \in \zeta^{\tilde{\mathcal{F}}}$ and based on the SVN-equivalence relation $\delta$ in a single-valued neutrosophic approximation space $(\tilde{\mathcal{F}}, \delta)$.

From (5) and (6), we obtain that $\tilde{\varrho}_{\left(\alpha_{n}\right)_{\delta}}(\omega) \leq \tilde{\varrho}_{\alpha_{n}}(\omega) \leq \tilde{\varrho}_{\left(\alpha_{n}\right)^{\delta}}(\omega), \tilde{\sigma}_{\left(\alpha_{n}\right)_{\delta}}(\omega) \geq$ $\tilde{\sigma}_{\alpha_{n}}(\omega) \geq \tilde{\sigma}_{\left(\alpha_{n}\right)^{\delta}}(\omega)$ and $\tilde{\zeta}_{\left(\alpha_{n}\right)_{\delta}}(\omega) \geq \tilde{\zeta}_{\alpha_{n}}(\omega) \geq \tilde{\zeta}_{\left(\alpha_{n}\right)^{\delta}}(\omega)$ for each $\alpha_{n} \in \zeta^{\tilde{\mathcal{F}}}$. Whenever $\left(\alpha_{n}\right)^{\delta}$ so that $\tilde{\varrho}_{\left(\alpha_{n}\right)^{\delta}}(\omega) \leq \tilde{\varrho}_{\left(\alpha_{n}\right)_{\delta}(\omega)}, \tilde{\sigma}_{\left(\alpha_{n}\right)^{\delta}}(\omega) \geq \tilde{\sigma}_{\left(\alpha_{n}\right)_{\delta}(\omega)}$ and $\tilde{\zeta}_{\left(\alpha_{n}\right)^{\delta}}(\omega) \geq \tilde{\zeta}_{\left(\alpha_{n}\right)_{\delta}(\omega)}$ we obtain that $\tilde{\varrho}_{\alpha_{n}}(\omega)=\tilde{\varrho}_{\left(\alpha_{n}\right)_{\delta}}(\omega)=\tilde{\varrho}_{\left(\alpha_{n}\right)^{\delta}}(\omega), \tilde{\sigma}_{\alpha_{n}}(\omega)=\tilde{\sigma}_{\left(\alpha_{n}\right)_{\delta}}(\omega)=\tilde{\sigma}_{\left(\alpha_{n}\right)^{\delta}}(\omega)$ and $\tilde{\zeta}_{\alpha_{n}}(\omega)=\tilde{\zeta}_{\left(\alpha_{n}\right)_{\delta}}(\omega)=\tilde{\zeta}_{\left(\alpha_{n}\right)^{\delta}}(\omega)$, and then from $(7)$, we obtain $\tilde{\varrho}_{\left(\alpha_{n}\right)^{B}}(\omega)=0, \tilde{\sigma}_{\left(\alpha_{n}\right)^{B}}(\omega)=1$ and $\tilde{\zeta}_{\left(\alpha_{n}\right)^{B}}(\omega)=1$. Otherwise, $\tilde{\varrho}_{\left(\alpha_{n}\right)^{B}}(\omega)=\left(\alpha_{n}\right)^{\delta} \wedge\left[\left(\alpha_{n}\right)_{\delta}\right]^{c}, \tilde{\sigma}_{\left(\alpha_{n}\right)^{B}}(\omega)=\left(\alpha_{n}\right)^{\delta} \vee\left[\left(\alpha_{n}\right)_{\delta}\right]^{c}$ and $\tilde{\zeta}_{\left(\alpha_{n}\right)^{B}}(\omega)=\left(\alpha_{n}\right)^{\delta} \vee\left[\left(\alpha_{n}\right)_{\delta}\right]^{c}$. .

Theorem 11. For any SVNS $\alpha_{n} \in \zeta^{\tilde{\mathcal{F}}}$ we find that (1) $\tilde{0}_{\delta}=\tilde{0}^{\delta}=\tilde{0}$ and $\tilde{1}_{\delta}=\tilde{1}^{\delta}=\tilde{1}$, 
(2) $\left(\alpha_{n} \vee \varepsilon_{n}\right)_{\delta} \geq\left(\alpha_{n}\right)_{\delta} \vee\left(\varepsilon_{n}\right)_{\delta}$

(3) $\left(\alpha_{n} \wedge \varepsilon_{n}\right)^{\delta} \leq\left(\alpha_{n}\right)^{\delta} \wedge\left(\varepsilon_{n}\right)^{\delta}$,

(4) $\alpha_{n} \leq \varepsilon_{n}$, implies that $\left(\alpha_{n}\right)^{\delta} \leq\left(\varepsilon_{n}\right)^{\delta}$ and $\left(\alpha_{n}\right)_{\delta} \leq\left(\varepsilon_{n}\right)_{\delta}$,

(5) $\left(\alpha_{n} \vee \varepsilon_{n}\right)^{\delta}=\left(\alpha_{n}\right)^{\delta} \vee\left(\varepsilon_{n}\right)^{\delta}$,

(6) $\left(\alpha_{n} \wedge \varepsilon_{n}\right)_{\delta}=\left(\alpha_{n}\right)_{\delta} \wedge\left(\varepsilon_{n}\right)_{\delta}$,

(7) $\left[\left(\alpha_{n}\right)^{\delta}\right]^{c}=\left(\left[\alpha_{n}\right]^{c}\right)_{\delta}$ and $\left[\left(\alpha_{n}\right)_{\delta}\right]^{c}=\left(\left[\alpha_{n}\right]^{c}\right)^{\delta}$,

(8) $\left[\left(\alpha_{n}\right)_{\delta}\right]^{\delta} \geq\left(\alpha_{n}\right)_{\delta}=\left[\left(\alpha_{n}\right)_{\delta}\right]_{\delta}$,

(9) $\left[\left(\alpha_{n}\right)^{\delta}\right]_{\delta} \leq\left(\alpha_{n}\right)^{\delta}=\left[\left(\alpha_{n}\right)^{\delta}\right]^{\delta}$.

Proof. Obvious.

Example 3. Let $\delta$ be an $S V N R$ on a set $\tilde{\mathcal{F}}=\left\{\omega_{1}, \omega_{2}, \omega_{3}\right\}$ as shown below.

\begin{tabular}{cccc}
\hline$\alpha_{n}$ & $\omega_{1}$ & $\omega_{2}$ & $\omega_{3}$ \\
\hline$\omega_{1}$ & $\langle 0,0,1\rangle$ & $\langle 0.3,0.1,0.6\rangle$ & $\langle 0.1,0,0.4\rangle$ \\
\hline$\omega_{2}$ & $\langle 0,0.2,0.4\rangle$ & $\langle 0.6,0.5,1\rangle$ & $\langle 0.6,0,1\rangle$ \\
\hline$\omega_{3}$ & $\langle 1,0,1\rangle$ & $\langle 1,0.5,1\rangle$ & $\langle 1,1,0\rangle$ \\
\hline
\end{tabular}

Assume that $\alpha_{n}=\langle(0.3,0.4,1),(0.5,0.2,0.2),(0.3,0.5,0.7)\rangle$. Then, the single-valued neutrosophic cosets are as follows:

$$
\begin{aligned}
& \tilde{\varrho}_{\left(\alpha_{n}\right)_{\delta}}\left(\omega_{1}\right)=\tilde{\varrho}_{\alpha_{n}}\left(\omega_{1}\right) \vee \bigwedge_{\left(\alpha_{n}\right)^{c}(\mu)>0, \mu \neq \omega_{1}} \tilde{\zeta}_{[\omega]}(\mu)=0.4 \\
& \tilde{\sigma}_{\left(\alpha_{n}\right)_{\delta}}\left(\omega_{1}\right)=\tilde{\sigma}_{\alpha_{n}}\left(\omega_{1}\right) \wedge \bigvee_{\left(\alpha_{n}\right)^{c}(\mu)>0, \mu \neq \omega_{1}}\left(1-\tilde{\sigma}_{\left[\omega_{1}\right]}\right)(\mu)=0.4 \\
& \tilde{\zeta}_{\left(\alpha_{n}\right)_{\delta}}\left(\omega_{1}\right)=\tilde{\zeta}_{\alpha_{n}}\left(\omega_{1}\right) \wedge{ }_{\left(\alpha_{n}\right)^{c}(\mu)>0, \mu \neq \omega_{1}} \tilde{\varrho}_{\left[\omega_{1}\right]}(\mu)=0
\end{aligned}
$$

Hence, $\left(\alpha_{n}\right)_{\delta}\left(\omega_{1}\right)=(0.4,0.4,0)$ and

$$
\begin{aligned}
& \tilde{\varrho}_{\left(\alpha_{n}\right)_{\delta}}\left(\omega_{2}\right)=\tilde{\varrho}_{\alpha_{n}}\left(\omega_{2}\right) \vee \bigwedge_{\left(\alpha_{n}\right)^{c}(\mu)>0, \mu \neq \omega_{2}} \tilde{\zeta}_{[\omega]}(\mu)=0.6 \\
& \tilde{\sigma}_{\left(\alpha_{n}\right)_{\delta}}\left(\omega_{2}\right)=\tilde{\sigma}_{\alpha_{n}}\left(\omega_{2}\right) \wedge \bigvee_{\left(\alpha_{n}\right)^{c}(\mu)>0, \mu \neq \omega_{2}}\left(1-\tilde{\sigma}_{\left[\omega_{2}\right]}\right)(\mu)=0.2 \\
& \tilde{\zeta}\left(\alpha_{n}\right)_{\delta}\left(\omega_{2}\right)=\tilde{\zeta} \alpha_{n}\left(\omega_{2}\right) \wedge \bigvee_{\left(\alpha_{n}\right)^{c}(\mu)>0, \mu \neq \omega_{2}} \tilde{\varrho}_{\left[\omega_{2}\right]}(\mu)=0.2
\end{aligned}
$$

Hence, $\left(\alpha_{n}\right)_{\delta}\left(\omega_{2}\right)=(0.6,0.2,0.2)$. Similarly, we can obtain $\left(\alpha_{n}\right)_{\delta}\left(\omega_{2}\right)=(0.4,0.5,0.7)$; therefore, $\left(\alpha_{n}\right)_{\delta}=\langle(0.4,0.4,0),(0.6,0.2,0.2),(0.4,0.5,0.7)\rangle,\left(\alpha_{n}\right)^{\delta}=\langle(0.3,0.4,0.4),(0.5,0.5$, $0.6),(0.3,0.5,0.7)\rangle$ and then $\left(\alpha_{n}\right)^{B}=\langle(0,0.6,0.4),(0.2,0.8,0.6),(0.3,0.5,0.7)\rangle$.

Definition 23. The single-valued neutrosophic approximation interior operator $\operatorname{int}_{\delta}^{\alpha_{n}}: \zeta^{\tilde{\mathcal{F}}} \rightarrow \zeta^{\tilde{\mathcal{F}}}$ is defined as follows:

$$
\operatorname{int}_{\delta}^{\alpha_{n}}\left(\varepsilon_{n}\right)=\left(\alpha_{n}\right)_{\delta} \wedge\left(\varepsilon_{n}\right)_{\delta}, \quad \forall \varepsilon_{n} \neq \tilde{1} \text { and } \operatorname{int}_{\delta}^{\alpha_{n}}(\tilde{1})=\tilde{1} .
$$

That is associated with an SVNS $\alpha_{n} \in \zeta^{\tilde{\mathcal{F}}}$ in a single-valued neutrosophic approximation $\operatorname{space}(\tilde{\mathcal{F}}, \delta)$.

Theorem 12. The following conditions are satisfied

(1) $\operatorname{int}_{\delta}^{\alpha_{n}}(\tilde{0})=\tilde{0}$,

(2) $\operatorname{int}_{\delta}^{\alpha_{n}}\left(\varepsilon_{n}\right) \leq \varepsilon_{n} \forall \varepsilon_{n} \in \zeta^{\tilde{\mathcal{F}}}$, 
(3) $\varepsilon_{n} \leq \pi_{n} \Rightarrow \operatorname{int}_{\delta}^{\alpha_{n}}\left(\varepsilon_{n}\right) \leq \operatorname{int}_{\delta}^{\alpha_{n}}\left(\pi_{n}\right), \forall \varepsilon_{n}, \pi_{n} \in \zeta^{\tilde{\mathcal{F}}}$,

(4) $\operatorname{int}_{\delta}^{\alpha_{n}}\left(\varepsilon_{n} \wedge \pi_{n}\right)=\operatorname{int}_{\delta}^{\alpha_{n}}\left(\varepsilon_{n}\right) \wedge \operatorname{int}_{\delta}^{\alpha_{n}}\left(\pi_{n}\right) \forall \varepsilon_{n}, \pi_{n} \in \zeta^{\tilde{\mathcal{F}}}$,

(5) $\operatorname{int}_{\delta}^{\alpha_{n}}\left(\operatorname{int}_{\delta}^{\alpha_{n}}\left(\varepsilon_{n}\right)\right)=\operatorname{int}_{\delta}^{\alpha_{n}}\left(\varepsilon_{n}\right) \forall \varepsilon_{n} \in \zeta^{\tilde{\mathcal{F}}}$.

Proof. For (1): $\operatorname{int}_{\delta}^{\alpha_{n}}\left(\varepsilon_{n}\right)=\left(\alpha_{n}\right)_{\delta} \wedge(\tilde{0})_{\delta}=\tilde{0}$.

For (2): $\operatorname{int}_{\delta}^{\alpha_{n}}\left(\varepsilon_{n}\right)=\left(\alpha_{n}\right)_{\delta} \wedge\left(\varepsilon_{n}\right)_{\delta} \leq\left(\alpha_{n}\right)_{\delta} \wedge \varepsilon_{n} \leq \varepsilon_{n}$.

For (3): $\varepsilon_{n} \leq \pi_{n}$ then $\left(\varepsilon_{n}\right)_{\delta} \leq\left(\pi_{n}\right)_{\delta} \Rightarrow \operatorname{int}_{\delta}^{\alpha_{n}}\left(\varepsilon_{n}\right) \leq \operatorname{int}_{\delta}^{\alpha_{n}}\left(\pi_{n}\right)$.

For (4): $\operatorname{int}_{\delta}^{\alpha_{n}}\left(\varepsilon_{n} \wedge \pi_{n}\right)=\left(\alpha_{n}\right)_{\delta} \wedge\left(\varepsilon_{n} \wedge \pi_{n}\right)_{\delta}=\left(\alpha_{n}\right)_{\delta} \wedge\left(\varepsilon_{n}\right)_{\delta} \wedge\left(\alpha_{n}\right)_{\delta} \wedge\left(\pi_{n}\right)_{\delta}=\operatorname{int}_{\delta}^{\alpha_{n}}\left(\varepsilon_{n}\right) \wedge$ $\operatorname{int}_{\delta}^{\alpha_{n}}\left(\pi_{n}\right)$.

For (5): Similarly to (4).

Thus, this is called a single-valued neutrosophic interior associated with $\alpha_{n}$ in the single-valued neutrosophic approximation space $(\tilde{\mathcal{F}}, \delta)$ generating a single-valued neutrosophic topology defined by:

$$
\omega_{\delta}^{\alpha_{n}}=\left\{\varepsilon_{n} \in \zeta^{\tilde{\mathcal{F}}}: \varepsilon_{n}=\operatorname{int}_{\delta}^{\alpha_{n}}\left(\varepsilon_{n}\right)\right\} .
$$

Definition 24. The single-valued neutrosophic approximation closure operator $\mathrm{Cl}_{\delta}^{\alpha_{n}}: \zeta^{\tilde{\mathcal{F}}} \rightarrow \zeta^{\tilde{\mathcal{F}}}$ is defined as follows:

$$
\mathrm{Cl}_{\delta}^{\alpha_{n}}\left(\varepsilon_{n}\right)=\left[\left(\alpha_{n}\right)_{\delta}\right]^{c} \vee\left(\varepsilon_{n}\right)^{\delta}, \quad \forall \varepsilon_{n} \neq \tilde{0} \text { and } \mathrm{Cl}_{\delta}^{\alpha_{n}}(\tilde{0})=\tilde{0} .
$$

Theorem 13. The single-valued neutrosophic approximation closure operator satisfies the following conditions:

(1) $\mathrm{Cl}_{\delta}^{\alpha_{n}}(\tilde{1})=\tilde{1} \forall \alpha_{n} \in \zeta^{\tilde{\mathcal{F}}}$,

(2) $\mathrm{Cl}_{\delta}^{\alpha_{n}}\left(\varepsilon_{n}\right) \geq \varepsilon_{n} \forall \varepsilon_{n} \in \zeta^{\tilde{\mathcal{F}}}$,

(3) $\varepsilon_{n} \leq \pi_{n} \Rightarrow \mathrm{Cl}_{\delta}^{\alpha_{n}}\left(\varepsilon_{n}\right) \leq \mathrm{Cl}_{\delta}^{\alpha_{n}}\left(\pi_{n}\right), \forall \varepsilon_{n}, \pi_{n} \in \zeta^{\tilde{\mathcal{F}}}$,

(4) $\mathrm{Cl}_{\delta}^{\alpha_{n}}\left(\varepsilon_{n} \wedge \pi_{n}\right)=\mathrm{Cl}_{\delta}^{\alpha_{n}}\left(\varepsilon_{n}\right) \wedge \mathrm{Cl}_{\delta}^{\alpha_{n}}\left(\pi_{n}\right)$ and $\mathrm{Cl}_{\delta}^{\alpha_{n}}\left(\varepsilon_{n} \vee \pi_{n}\right)=\mathrm{Cl}_{\delta}^{\alpha_{n}}\left(\varepsilon_{n}\right) \vee \mathrm{Cl}_{\delta}^{\alpha_{n}}\left(\pi_{n}\right) \forall \varepsilon_{n}, \pi_{n} \in$ $\zeta^{\tilde{\mathcal{F}}}$,

(5) $\mathrm{Cl}_{\delta}^{\alpha_{n}}\left(\mathrm{Cl}_{\delta}^{\alpha_{n}}\left(\varepsilon_{n}\right)\right)=\mathrm{Cl}_{\delta}^{\alpha_{n}}\left(\varepsilon_{n}\right) \forall \varepsilon_{n} \in \zeta^{\tilde{\mathcal{F}}}$.

Proof. Similar to the proof of Theorem 12.

\section{Conclusions}

In this paper, we defined the single-valued neutrosophic semi-closure space (SVNSCS). It has been proven that every $\tau_{\mathbb{S C}}^{\tau \tilde{\rho} \widetilde{\sigma} \tilde{F}}$ is a single-valued neutrosophic ideal topological space on $\mathcal{F}$. It has also been proven that every $\mathbb{S C}_{\mathbb{S} \mathbb{C}}^{\tau \tilde{\sigma} \tilde{F}}$ is finer than $\mathbb{S} \mathbb{C}$ (see Theorem 2). In addition,

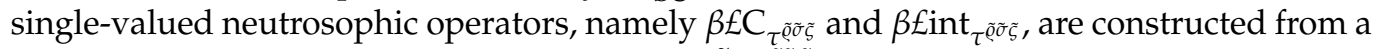
single-valued neutrosophic topological space $\left(\tilde{\mathcal{F}}, \tau^{\hat{\tilde{\rho}} \tilde{\sigma} \tilde{s}}\right)$ ) (see Theorem 5). Next, the concepts of a single-valued neutrosophic almost $\beta €$-continuous, single-valued neutrosophic faintly $\beta £$-continuous and single-valued neutrosophic weakly $\beta £$-continuous based on a singlevalued neutrosophic ideal $£^{\tilde{\rho} \tilde{\sigma} \tilde{S}}$ were introduced and studied (see Theorems $\left.6-8\right)$. Finally, we introduced the single-valued neutrosophic sets $\left(\alpha_{n}\right)_{\delta},\left(\alpha_{n}\right)^{\delta},\left(\alpha_{n}\right)^{B}$ for a single-valued neutrosophic set $\alpha_{n}$ that explains the single-valued neutrosophic roughness of the singlevalued neutrosophic set $\alpha_{n}$. We introduced the notion of single-valued neutrosophic approximation space and the related single-valued neutrosophic topology.

\section{Discussion for Further Works}

The theories that were used in this article could be extended to study some similar notions in the neutrosophic metric topological spaces.

Funding: This research received no external funding.

Institutional Review Board Statement: Not applicable. 
Informed Consent Statement: Not applicable.

Data Availability Statement: Not applicable.

Acknowledgments: The author would like to express his sincere thanks to the Referees for their valuable comments and suggestions, which led to the improvement of this work.

Conflicts of Interest: The author declares that there is no conflict of interest regarding the publication of this manuscript.

\section{References}

1. Smarandache, F. A Unifying Field in Logics, Neutrosophy: Neutrosophic Probability, Set and Logic; American Research Press: Rehoboth, NM, USA, 1999.

2. Bakbak, D.; Uluçay, V.; Şahin, M. Neutrosophic Soft Expert Multiset and Their Application to Multiple Criteria Decision Making. Symmetry 2018, 7, 50 .

3. Mishra, K.; Kandasamy, I.; Kandasamy, V.; Smarandache, F. A Novel Framework Using Neutrosophy for Integrated Speech and Text Sentiment Analysis. Symmetry 2020, 12, 1715. [CrossRef]

4. El Naschie, M.S. Quantum gravity, Clifford algebras, fuzzy set theory and the fundamental constants of nature. Chaos Solitons Fractals 2004. 20, 437-450. [CrossRef]

5. El Naschie, M.S. On the unification of heterotic strings, M theory and $\varepsilon^{\infty}$ theory. Chaos Solitons Fractals 2000 11, $2397-2408$. [CrossRef]

6. Salama, A.A.; Alblowi, S.A. Neutrosophic set and neutrosophic topological spaces. IOSR J. Math. 2012, 3, 31-35. [CrossRef]

7. Salama, A.A.; Smarandache, F.; Kroumov, V. Neutrosophic crisp sets and neutrosophic crisp topological spaces. Neutrosophic Sets Syst. 2014, 2, 25-30.

8. Salama, A.A.; Smarandache, F. Neutrosophic Crisp Set Theory; Educational Publisher: Columbus, OH, USA, 2015.

9. Chang, C.L. Fuzzy Topological Spaces. J. Math. Anal. Appl. 1968, 24, 182-190 [CrossRef]

10. Hur, K.; Lim, P.K.; Lee, J.G.; Kim, J. The category of neutrosophic sets. Neutrosophic Sets Syst. 2016, 14, 12-20.

11. Hur, K.; Lim, P.K.; Lee, J.G.; Kim, J. The category of neutrosophic crisp sets. Ann. Fuzzy Math. Inform. 2017, 14, 43-54. [CrossRef]

12. Smarandache, F. A Unifying Field in Logics: Neutrosophic Logic. Neutrosophy, Neutrosophic Set, Neutrosophic Probability and Statistics, 6th ed.; InfoLearnQuest: Ann Arbor, MI, USA, 2007. Available online: http:/ / fs.gallup.unm.edu/eBook-neutrosophics6.pdf (accessed on 10 February 2019).

13. Šostak, A. On a fuzzy topological structure. In Circolo Matematico di Palermo, Palermo; Rendiconti del Circolo Matematico di Palermo, Proceedings of the 13th Winter School on Abstract Analysis, Section of Topology, Srni, Czech Republic, 5-12 January 1985; Circolo Matematico di Palermo: Palermo, Italy, 1985; pp. 89-103.

14. Fang, J.M.; Yue, Y.L. Base and Subbase in I-fuzzy Topological Spaces. J. Math. Res. Expos. 2006, 26, 89-95.

15. Zahran, A.M.; Abbas, S.E.; Abd El-baki, S.A.; Saber, Y.M. Decomposition of fuzzy continuity and fuzzy deal continuity via fuzzy idealization. Chaos Solitons Fractals 2009, 42, 3064-3077. [CrossRef]

16. Saber, Y.M.; Abdel-Sattar, M.A. Ideals on fuzzy topological spaces. Appl. Math. Sci. 2014, 8, 1667-1691. [CrossRef]

17. Wang, H.; Smarandache, F.; Zhang, Y.Q.; Sunderraman, R. Single valued neutrosophic sets. Multispace Multistruct. 2010, 4, 410-413.

18. El-Gayyar, M. Smooth neutrosophic topological spaces. Neutrosophic Sets Syst. 2016, 65, 65-72.

19. Kim, J.; Smarandache, F.; Lee, J.G.; Hur, K. Ordinary Single Valued Neutrosophic Topological Spaces. Symmetry 2019, 11, 1075 [CrossRef]

20. Al Shumrani, M.A.; Gulisten, M.; Smarandache, F. Further theory of neutrosophic triplet topology and applications. Symmetry 2020, 12, 1207. [CrossRef]

21. Saber, Y.; Alsharari, F.; Smarandache, F. On single-valued neutrsophic ideals in Šostak's sense. Symmetry 2020, 12, 193. [CrossRef]

22. Saber, Y.; Alsharari, F.; Smarandache, F. Connectedness and Stratification of Single-Valued Neutrosophic Topological Spaces. Symmetry 2020, 12, 1464. [CrossRef]

23. Alsharari, F. £-Single Valued Extremally Disconnected Ideal Neutrosophic Topological Spaces. Symmetry 2021, 13, 53. [CrossRef]

24. Alsharari, F. Decomposition of Single-Valued Neutrosophic Ideal Continuity via Fuzzy Idealization. Neutrosophic Sets Syst. 2020, 38, 145-163

25. Alsharari, F.; Saber, Y.M. $\mathcal{G} \Theta_{\tau_{i}}^{\star \tau_{j}}$-Fuzzy closure operator. New Math. Nat. Comput. 2020, 16, 123-141. [CrossRef]

26. Yang, H.L.; Guo, Z.L.; Liao, X. On single valued neutrosophic relations. J. Intell. Fuzzy Syst. 2016, 30, 1045-1056. [CrossRef]

27. Kim, J.; Lim, P.K.; Lee, J.G.; Hur, K. Single valued neutrosophic relations. Ann. Fuzzy Math. Inform. 2018, 16, $201-221$. 